\title{
Design Considerations for a Portable Raman Probe Spectrometer for Field Forensics
}

\author{
James F. Kelly, Thomas A. Blake, Bruce E. Bernacki, and Timothy J. Johnson
}

Pacific Northwest National Laboratory, P. O. Box 999, Mail Stop K8-88, Richland, WA 99352, USA

Correspondence should be addressed to Thomas A. Blake, ta.blake@pnnl.gov

Received 20 February 2012; Revised 4 June 2012; Accepted 12 June 2012

Academic Editor: Steven Christesen

Copyright () 2012 James F. Kelly et al. This is an open access article distributed under the Creative Commons Attribution License, which permits unrestricted use, distribution, and reproduction in any medium, provided the original work is properly cited.

\begin{abstract}
Raman spectroscopy has been shown to be a viable method for explosives detection. Currently most forensic Raman systems are either large, powerful instruments for laboratory experiments or handheld instruments for in situ point detection. We have chosen to examine the performance of certain benchtop Raman probe systems with the goal of developing an inexpensive, portable system that could be used to operate in a field forensics laboratory to examine explosives-related residues or samples. To this end, a rugged, low distortion line imaging dispersive Raman spectrograph was configured to work at $830 \mathrm{~nm}$ laser excitation and was used to determine whether the composition of thin films of plastic explosives or small (e.g., $\leq 10 \mu \mathrm{m})$ particles of RDX or other explosives or oxidizers can be detected, identified, and quantified in the field. With $300 \mathrm{~mW}$ excitation energy, concentrations of RDX and PETN can be detected and reconstructed in the case of thin Semtex smears, but further work is needed to push detection limits of areal dosages to the $\sim 1 \mu \mathrm{g} / \mathrm{cm}^{2}$ level. We describe the performance of several probe/spectrograph combinations and show preliminary data for particle detection, calibration and detection linearity for mixed compounds, and so forth.
\end{abstract}

\section{Introduction}

There is an ever increasing need for inexpensive and reliable detection of trace chemicals on surfaces for security and military applications [1]. In the homeland security and military arenas, chemicals such as explosives, and chemical and biological warfare agents all continue to be potential threats. In the last decade, Raman spectroscopy, particularly in the visible and near-infrared (NIR) wavelengths, has emerged as a method to detect and identify materials in a rapid and inexpensive manner [2]. One compelling motivation for the use of Raman techniques is that the spectra are more straightforward to analyze than infrared spectra, due in part to narrower intrinsic linewidths. Often the analyte Raman spectra can be simply and quickly interpreted in terms of peak height and/or peak area comparisons with an appropriate database [2]. Component concentrations of plastic explosives, for example, appear to be treatable as simple linear superpositions of spectra, and quantifications can sometimes be done to about the $1 \%$ level of contamination with bulk samples [3]. As a consequence, identifying the provenance of materials, especially explosives, becomes more straightforward. Raman spectroscopy has transitioned from the research laboratory to a field analytical technique since it can rapidly detect and identify, with the use of an appropriate spectral database, thousands of chemicals including weapons of mass destruction materials [2]. Each year the systems, especially those developed to study residual energetic materials, continue to evolve, becoming smaller, more reliable and lower in cost $[4,5]$.

Most of the modern portable systems used in the study of explosives and their precursors use near-infrared lasers for excitation to reduce the likelihood of fluorescence, heating, or other phenomena that can overwhelm the Raman signal of the explosives. Such Raman sensors have been shown to be particularly useful for identifying chemical compounds associated with explosives, both pre- and postblast. Indeed, handheld Raman sensors are already in use by military forces for such applications [5]. At the other extreme are large and expensive laboratory Raman microscope systems that now offer imaging with excellent spatial resolution and Raman sensitivity, but are not generally amenable to field use [6, 7]. These confocal spectral imagers (CSIs) are up to the task of analyzing small spots with excellent magnification 
and chemical specificity, but they are designed around microscope systems of both high numerical aperture (NA) and high magnification, so necessitate careful imaging with limited depth of field (DoF). Field work requires much less magnification to improve DoF and to increase the field of view for wider area surveys. However, a reasonably high NA (higher NA, higher throughput) should be maintained so that fast analyses can be achieved over larger areas approaching 1 by $2 \mathrm{~cm}^{2}$. Our work suggests that Offnerbased dispersive spectrographs using macro-lens coupling designs will permit wide line laser-illumination, yet offer efficient recovery of Raman backscattering that can help view large areas at low magnification. The relative throughput of signal recovery was tested with low magnification and modest NA Raman probes to verify that analysis of chemical residues over uneven or variegated surfaces is possible, potentially to single particles of RDX with sizes $\approx 10 \mu \mathrm{m}$. This is the cutoff target particle size recommended for study of residue samples, because repeated manipulation of the particles causes fragmentation that leads to the greatest cumulative increase of mass density above this size [8].

The approach recommended for best signal recovery of large area surveys of such small particles is analogous to photoemission microscopy [9]. It is feasible to use low magnification macroelement imaging systems that can optimize light throughput while achieving the spatial resolution of the CCD pixel size over a much wider full field of view supported by the lens and camera system. A patent by Kain for full field microimaging with optimal throughput describes a low magnification epi-illumination technique that would be compatible for line-illumination Raman imaging, which could significantly reduce the DoF constraints for field work [10].

Our objective is to build a prototype Raman instrument that would fill the technology gap between the handheld sensors and the research grade, confocal Raman spectrometers. Such an instrument would be field portable, fit on a $2^{\prime} \times 2^{\prime}$ bread board, run on standard AC power, have an aircooled CCD array that can operate in a variety of environmental conditions, could detect, identify, and image chemical particles down to $10 \mu \mathrm{m}$ in size or spots of chemical thin films with an areal dosage of $1 \mu \mathrm{g} / \mathrm{cm}^{2}$ on forensic samples with irregular surfaces, and can be operated with minimal training. Noncontact evaluation of suspect materials could be done within a few centimeters of their surfaces. In this paper we describe preliminary work in testing performance concepts for such an imaging instrument by examining the performance of two commercial, off-the-shelf Raman probe spectrometers provided as demo instruments. From this work we devised and tested the performance of a spectrograph/CCD detector combination that we selected as the heart of what will become our imaging instrument. Fourier transform (FT) Raman database spectra are used for comparative Raman spectral analysis and Stokes shift calibration purposes; these spectra are described, as needed, in the text. Experimental results with respect to resolution, detection sensitivity, calibration, and detection linearity for mixed compounds are discussed. Based on these results, we then consider design improvements for a proposed Raman line imager without recourse to confocal imaging and tight depth of field restraints. A specific optical design of the line illumination-image relay optics is also presented.

\section{Preliminary Operational and Design Considerations}

The first objective of this work was the evaluation of a simpler or more robust Raman system based on the recent techniques of making optically fast (low F/\#) stigmatic imaging grating spectrographs. This entails several considerations as detailed below. Figure 1 shows the basic architecture we were striving to devise, based on an Offner-Dyson design [11]. It would be a line illumination variant of the confocal spectral imaging systems that Renishaw first devised [6] but use an OffnerDyson spectrograph as the dispersive spectral engine to enhance the pixel sampling resolution interval. (For the Headwall spectrograph described below, the dispersion is either 2.37 or $1.64 \mathrm{~cm}^{-1}$ per pixel assuming a $26 \mu \mathrm{m}$ array spacing and depending on the input port used.) Such a design is ideal for making hyperspectral line imagers with low F/\#. For the best efficiency with a commercial F/2.8 spectrograph, an illumination macrolens of $\mathrm{F} / 1.4$ is recommended with an F/2.8 relay macro-lens to achieve optimal throughput and a modest magnification of 2 to match with a CCD array pixel size of $26 \mu \mathrm{m}$. The operational goal is to simplify the input optics and detect direct backscattering with the same focusing optic (epi-illumination) and use a line source of laser light to reduce the specific intensity of focused excitation light on a target, but still be able to integrate signals with overall greater average total power. Following McCreery's suggestions [12] of using line imaging with rotating samples to enhance sample averaging, it was expected that the system could be a dual-use portable chemical imager. It could be field-hardened to serve a mobile laboratory, but also capable of doing in situ studies of stains.

2.1. Preliminary Considerations: Excitation Wavelength and Power. For Raman spectroscopy, the optimal excitation wavelength is an application-dependent tradeoff between increased signal versus interference, mostly from fluorescence, but sometimes from grey-body thermal emission of dyestuffs added to the explosives $[13,14]$. Shifting to shorter excitation wavelengths gains signal according to the " $v^{4}$ " factor. (In fact, if a power-detecting element is used the appropriate expression is $\left(\nu_{0}-v_{k}\right)^{4}$ whereas if a photoncounting device is used, the response term is $v_{0}\left(v_{0}-v_{k}\right)^{3}$ where $\nu_{0}$ is the laser frequency and $\nu_{k}$ the vibrational mode frequency [15].) Shifting to shorter wavelengths, however, often induces molecular fluorescence whereby the laser light is absorbed and reemitted via an electronic transition that does not involve a change in the electronic spin quantum. Fluorescence signals are typically broad and featureless and often obscure the Raman signal. In those cases, shifting to longer wavelength excitation can be advantageous, though signal is sacrificed.

Akhavan was the first to show that explosives such as Semtex formulations, which contain significant dye additives and fluorescing compounds such as oils and organic binders, 


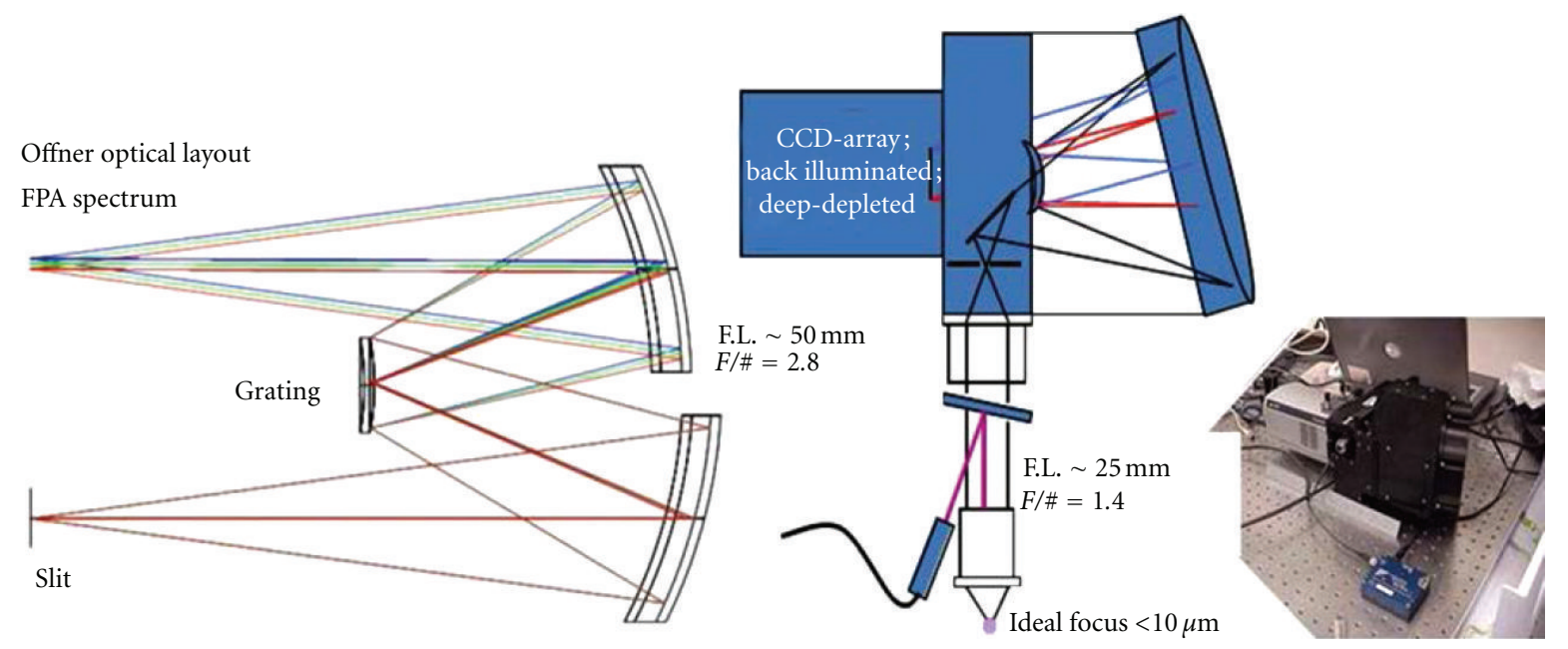

FIGURE 1: The ray trace of a Raman line imaging spectrograph using an Offner convex grating and concentric mirror is shown in the figure on the left. The object plane and image plane are identical. The figure in the middle shows a notional Offner-Dyson layout where the collimating and focusing mirror are intentionally tilted to transfer the rays onto the convex grating so that the resulting image plane is below the input slit plane. A photograph of the Headwall/Andor demo system is shown on the right.

can be properly analyzed using $1064 \mathrm{~nm}$ excitation and a Fourier-transform (FT) Raman spectrometer [16, 17]. In that seminal work, it was shown that the relative proportions of RDX and PETN could be discerned with an FT-Raman instrument with a resolution of $6 \mathrm{~cm}^{-1}$; however, sufficient signal-to-noise ratio (SNR) required fairly high excitation power $(\geq 700 \mathrm{~mW})$ and rather long averaging of $\sim 100$ to 200 scans ( 6 to 12 minute averaging times). Later work with multichannel dispersive spectrographs has shown similar benefits of using longer excitation wavelengths to help interrogate and assess Semtex [4]. The most involved studies of the tradeoffs of excitation wavelength and resolution carried out to understand issues of spectral interference in Semtex formulations were performed by the group of Lewis et al. [18-22].

Lewis et al. were the first to suggest that for certain Semtex species intermediate NIR wavelengths shorter than $1064 \mathrm{~nm}$ may be useful for interrogation with lowered fluorescence $[18,22]$. At the outset of our work, it was assumed that rather than use the $785 \mathrm{~nm}$ wavelength, which was found to be problematic for Semtex by Lewis et al. the preferable excitation should be an even longer wavelength provided by stabilized $1064 \mathrm{~nm}$ Nd:YAG lasers. However, shifting to this wavelength complicates the detection of the longer Stokes shifted Raman lines, which are consequently shifted beyond the reach of Si-based CCD technologies. InGaAs arrays and more exotic photocathode-based imagers are needed to see the stronger population-filled Stokes lines with $1064 \mathrm{~nm}$ excitation. Using Stokes emission exclusively would therefore greatly increase the cost of the imaging array, and also add the penalty of greater thermoelectric cooling of the array to minimize dark currents. The Lewis' were able to show that by using the much weaker anti-Stokes emissions from $1064 \mathrm{~nm}$ excitation they could observe the RDX and PETN components of Semtex with greatly reduced fluorescence [20]. Their F/1.8 dispersive spectrographs were optically fast and allowed nominally undistorted summing of the signal along the middle of the slit length using SiCCD array technology. Smile and keystone distortions in the Kaiser Optical Systems, Inc. transmissive spectrographs are sufficiently corrected to cleanly sum 4 to 8 pixel-tall signals produced by standard Raman probes [23, 24]. With a $26 \mu \mathrm{m}$ pixel pitch of traditional spectroscopy-grade imaging arrays, a Raman probe's collection fiber, typically either 95 or $200 \mu \mathrm{m}$ diameter, will produce a series of vertically offset spectral lines located symmetrically about the central optical axis. Spatial summing gives a direct means to statistically average many coregistered spectra improving their relative statistical deviation $[12,25]$.

While the Lewis' work was impressive and would allow bulk analyses of Semtex, it is not the only consideration to achieve sensitive detection of spatially extended, low areal dose residues. However, it should be noted that the Headwall Raman Explorer instrument made for $785 \mathrm{~nm}$ excitation could be easily adapted with an Si-CCD to study the antiStokes Raman lines with $1064 \mathrm{~nm}$ Raman probes by using the second input port (Port 2) feature that handles light from $1144 \mathrm{~nm}$ to $955 \mathrm{~nm}$. This allows $\sim 650 \mathrm{~cm}^{-1}$ of the antiStokes light to be analyzed with an average reciprocal linear dispersion of $1.64 \mathrm{~cm}^{-1}$ per pixel element assuming a $26 \mu \mathrm{m}$ array pixel pitch. Our Fourier transform (FT) Raman spectra using $1064 \mathrm{~nm}$ excitation-recorded to create a database for explosives-related compounds-indicate that the important thermally populated lattice modes of most solid explosives could largely be seen with this approach. The performance of the Headwall Port 2 option using a $1064 \mathrm{~nm}$ Raman with a notch/edge filtered probe that could transmit the anti-Stokes signals was not tested because of expense and availability of suitable probes. Instead we worked with the commercial $785 \mathrm{~nm}$ Headwall instrument, but used an edge filtered $830 \mathrm{~nm}$ Raman probe to test for signal sensitivity and confirm basic resolution tradeoffs with probe NA and fiber 
core size. Our laboratory confirmed that moving from 785 to $830 \mathrm{~nm}$ did in fact reduce the fluorescence as posited by Lewis et al. $[18,22]$. Based on all these various considerations, it was determined that for our prototype, $830 \mathrm{~nm}$ was the wavelength of choice.

In the development of a field portable Raman line imager, the cost is determined chiefly by the detector array, then the spectrograph, and next the probe-collection optics, and finally the laser source. Modern NIR distributed feedback (DFB) and grating feedback stabilized diode lasers have progressed so dramatically in the last several years that it is feasible to get $>300 \mathrm{~mW}$ stabilized continuous wave powers with laser linewidth $<1.0 \mathrm{~cm}^{-1}$ at 785 or $830 \mathrm{~nm}$ for reasonable cost. With such high available laser fluences, it now makes sense to use line imaging epi-illumination (collection objective is also the illumination objective) to reduce the power density on the target. Similarly, current-art confocal spectral imagers are no longer limited by the lower brightness (specific intensity-photons $\mathrm{s}^{-1} \mathrm{~cm}^{-2} \mathrm{sr}^{-1}$ ) afforded by traditional gas-discharge laser sources. Ramsey points out that line-focused Raman spectrometers can greatly exceed the performance of a confocal based Raman imager (but this assumes a neatly distributed sample) and, that monolayers of materials can be analyzed in the laboratory with such systems and modern CCD arrays [25]. Early work using a Raman line imaging spectrometer to study heterogeneously distributed explosives specifically as fingerprint contamination is described in [7], but it was limited to using a microscope delivery-collection system of small depth of field (DoF) and field of view over which scanning was performed.

\subsection{Preliminary Considerations: Spectroscopic Resolution.} Figure 2 below illustrates a comparison of spectral resolution for the strong Raman scattering material sodium chlorate. In spectroscopic work, a most important criterion for discerning distinctive narrow peaks in a large background of broad incoherent signal is resolution. The finer points of the tradeoffs of this in the course of doing confocal spectral imaging (CSI) are discussed for various slit spectrograph architectures in $[25,26]$. Typically, sharp peaks will show up on top of the diffuse random scatter/fluorescence with high spectral resolution instruments, but as resolution is reduced, fluctuations of the diffuse surface scatter or background fluorescence will overwhelm the narrow weaker signal peaks. More subtle methods of second derivative testing of the slope changes or methods of optimal filtering or advanced multivariate spectral analyses must be utilized to see effects of the nearly buried peaks. These approaches use higher signal-tonoise ratios (SNR) to determine the peak frequencies found mixed within the broad contours of diffuse scatter. Based on the study of several key energetic materials such as the data displayed in Figure 2, it was empirically determined that a resolution of better than 10 to $12 \mathrm{~cm}^{-1}$ (FWHM) would be needed to attain the desired specificity for our application, though this parameter is tightly linked to the numerical aperture and spot size considerations discussed next.

2.3. Preliminary Considerations: Numerical Aperture. High numerical aperture (NA) at the collection optic is paramount



Figure 2: Comparison of nearly blended Raman peaks of sodium chlorate (scaled to match). The sodium chlorate Raman spectrum recorded with the Headwall/Andor demo grating spectrograph and two-dimensional silicon-CCD is shown in green; the spectrum recorded with the DeltaNu Advantage demo grating spectrograph and InGaAs two-dimensional CCD is shown in red; the Bruker FT-Raman spectrum with nitrogen-cooled Ge detector is shown in blue. Data from the grating spectrographs were acquired in $1 \mathrm{~s}$ full integration time. Inset shows an expanded area of the residual scattered light.

when it comes to detecting weak fluorescence or Raman signals with high optical efficiency. Throughput efficiency scales strongly with the solid angle of acceptance $\Omega$ of the sample objective. In the case of circular epi-illumination this solid angle is given as $\Omega=2 \pi\left[1-\sqrt{ }\left\{1-(\mathrm{NA})^{2}\right\}\right]$, so overall efficiency for circular epi-illumination (without considering retro-reflection effects from irregularities in highly reflective surfaces) will be $\Omega / 4 \pi$. Table 1 lists some common values of F/\# (proportional to 1/NA for F/\#'s $\gtrsim 2$ ) of good commercial telecentric macro lenses. This range of macro lenses would be appropriate for resolving particulates (without immersion) down to a 5 to $10 \mu \mathrm{m}$ spot size. They would offer a decent DoF and wider field of view than microscope objectives designed for high magnification and provide considerable cost reduction for developing longer wavelength Raman imagers. If the NA of the illumination-collection objective is greatly increased from that of standard Raman probes (NA $\sim 0.22$ to 0.27 ) then Raman signal will be significantly increased by using such free-space microimaging with epiillumination. In the work reported here we used a simple fiber probe with nominal $100 \mu \mathrm{m}$ probe area, and similar depth of field. This adds collateral signal from the substrate onto each vertical pixel when trying to resolve small particles and so is not the best way to operate with thin smears where the particulates may segregate.

For confocal spectral imagers (CSIs), the speed of signal acquisition is limited by the practical magnification, $\mathrm{M}$, and the NA of the optics to spatially resolve very small grains. If high spatial resolution is retained near the diffraction limit (half-width) of $0.61 \cdot \lambda / \mathrm{NA}$, where NA is the numerical 
TABLE 1: Comparison of available NIR macro lenses as a function of F/\# and their corresponding NA, collection solid angle $\Omega$ and, efficiency (assuming isotropic Raman scatter).

\begin{tabular}{ccccc}
\hline $\mathrm{F} / \#$ & $\mathrm{NA}^{\mathrm{a}}$ & $\Theta_{1 / 2}{ }^{\mathrm{b}}(\mathrm{deg})$. & $\Omega(\mathrm{sr})$ & $\Omega / 4 \pi(\%)$ \\
\hline 2.8 & 0.1758 & 10.12 & $2 \pi(0.015574)$ & 0.78 \\
& & & 0.09785 & \\
1.8 & 0.2676 & 15.52 & $2 \pi(0.03647)$ & 1.8 \\
& & & 0.2291 & \\
1.4 & 0.3363 & 19.65 & $2 \pi(0.05825)$ & 2.9 \\
& & & 0.366 & \\
1.14 & 0.4017 & 23.68 & $2 \pi(0.08423)$ & 4.2 \\
& & & 0.5292 & \\
1 & 0.4472 & 26.57 & $2 \pi(0.1056)$ & 5.3 \\
\hline
\end{tabular}

${ }^{\mathrm{a}} \mathrm{NA}=\sin \left\{\tan ^{-1}[1 /(2 \cdot \mathrm{F} / \#)]\right\}$.

${ }^{\mathrm{b}} \Theta_{1 / 2}=\tan ^{-1}[1 /(2 \cdot \mathrm{F} / \#)]$.

aperture of the collection objective, and high magnification, $\mathrm{M}$, is retained for visualizing the explosive grains with CCD arrays, then throughput is greatly limited. In the case of epiillumination of the sample with a bore-sighted laser that induces Raman backscatter (and fluorescence), signal will scale as $(\mathrm{NA})^{4} / \mathrm{M}^{2}$ for a $2 \mathrm{D}$ scanning confocal system [27], such as the Renishaw system [6]. Increasing laser power will help, but the targets could be damaged or transformed under localized heating once focused intensities approach $1 \mathrm{MW} / \mathrm{cm}^{2}$ - even for uncolored scatterers; work with the Renishaw system typically holds the laser fluence below $0.25 \mathrm{MW} / \mathrm{cm}^{2}$ [6]. Going to a line imager, the collection efficiency of a high numerical aperture objective can be maintained, and if imaging along the slit is desired, then brightness reductions will scale less severely with magnification, $\mathrm{M}$, as $(\mathrm{NA})^{4} / \mathrm{M}$, because throughput along the slit will be directly summed as separate tracks. Increasing SNR, gained largely by statistical averaging of many $(N)$ independent spectra, will be improved slowly as $\sqrt{ } N$, but modern CCD arrays permit rapid direct summing by a full vertical binning feature. McCreery suggests that this feature of improved étendue (throughput) is indeed plausible, although an exact numerical confirmation has not, to our knowledge, yet been done with changing magnifications $[12,25]$. Based on the above considerations, an $\mathrm{F} / \#$ in the 1.5 to 2.0 range was deemed optimal in terms of increased optical throughput without sacrificing too much spatial resolution, but such a fast Offner system was not available specifically for the Raman excitation wavelength at which we wanted to work $(830 \mathrm{~nm})$.

\section{Experimental}

With the above considerations borne in mind, we began the investigation by considering already-available commercial Raman spectrometers to determine if the Raman spectra remained more representative of the bulk peaks as substrates were changed or residues were diluted. The working assumption was that Raman scattering of near-IR lasers, being a strong scattering process like Rayleigh scattering $\left(\sim v^{4}\right)$, will largely not penetrate to the interface as readily as deeper IR, which seems to probe the interfacial and/or morphological effects with higher sensitivity. Based on the considerations to use a high étendue spectrometer of the Offner design, we found that Headwall has devised a number of industrial grade spectrographs for both hyperspectral imaging in the near-IR, and for Raman line imaging applications [28]. These designs are highly corrected for astigmatism, spherical aberration, coma and field curvature. Astigmatism is the strongest aberration to reduce spatial signal summing and scales quadratically with both the NA of the spectrograph and with the angle of off-axis rays from the centerline of the spectrograph [26]. The Headwall Raman Explorer (tailored for $785 \mathrm{~nm}$ excitation) typically reduces the distortions, known as smile and keystone, resulting from astigmatism and other aberrations to $<0.1 \%$ [28]. While it may not be as ideal for trace residue analysis as the multichannel FTRaman spectrometer devised by Zhao and McCreery [29], it should serve well as a multi-track imager (using multiple Raman probes) or as a free-space optical line imaging Raman spectrograph.

Before assembling our own Raman probe instrument for testing we acquired two demonstration systems. One was from Andor Technology, Inc. which used an Andor Newton DU-920N-BRD, $256(\mathrm{v}) \times 1024(\mathrm{~h})(26 \mu \mathrm{m}$ pixel pitch $)$ spectroscopic grade Si-CCD with deep-depleted option that was back illuminated to reduce étalon fringing. The standard software package was used to acquire signals, and could display spectral curves, or be used to study the focal plane array (FPA) intensity grid. Rapid update of line averaged results was possible with the Full Vertical Binning option, which was used in collecting all the spectral data shown for this system. The optimal Nyquist limit for a $25 \mu \mathrm{m}$ slit width (Headwall \#1 slit) would require a $13 \mu \mathrm{m}$ pixel pitch CCD array to have at least three pixel samples at the baseline of a Raman peak. The results shown in this report were obtained with a $25 \mu \mathrm{m}$ slit width and $26 \mu \mathrm{m}$ pitch CCD, so desired resolution is degraded about $50 \%$. The demo camera was mated to a Headwall Photonics Raman Explorer $785(\mathrm{~F} / \#=2.8)$. The $6 \mathrm{~mm} \times 25 \mu \mathrm{m}$ slit was used and the demo spectrograph had a 200 lines $/ \mathrm{mm}$ concave grating. An EnWave Optronics, Inc. Raman probe with a smaller excitation/collection core diameter (95 $\mu \mathrm{m}$ diameter excitation fiber/100 $\mu \mathrm{m}$ diameter collection fiber) probe was used in our early evaluation work. The excitation source was a $785 \mathrm{~nm}, 40 \mathrm{~mW}$ Innovative Photonic Solutions, Inc. solid state external cavity laser. The EnWave probe has NA $\approx 0.22$ and thus overfilled the Headwall spectrograph. We will refer to this system as the Headwall/Andor demo.

The second demo instrument was a DeltaNu Advantage 1064 that uses $1064 \mathrm{~nm}$ excitation, an Intevac, Inc. MOSIR 950 InGaAs-based photocathode amplified detector with $1024 \times 256,26 \mu \mathrm{m}$ pixels, and a free-space lens system to illuminate and collect the laser and scattered light. The effective resolution of this system is approximately $15 \mathrm{~cm}^{-1}$. Our performance with this system to study Semtex samples was quite comparable to data reported by Vitek et al. [30] We will refer to this system as the DeltaNu demo. 
Figure 2 shows an example of an early comparison of the two demo systems against FT-Raman data taken at $4 \mathrm{~cm}^{-1}$ resolution for the case of a strong Raman scattering salt, sodium chlorate. For certain comparisons, we have juxtaposed spectra from these dispersive systems with high resolution laboratory measurements of bulk materials made with a Fourier transform (FT) Raman spectrometer. The FT-Raman instrument was a Bruker $66 \mathrm{v}$ that used a $1064 \mathrm{~nm}$ YAG laser excitation and liquid nitrogen-cooled Ge detector [15]. Apart from the FT-Raman spectrum of sodium chlorate shown in Figure 2, which was recorded with $4 \mathrm{~cm}^{-1}$ resolution, the remaining FT-Raman data shown in this paper were recorded at $2 \mathrm{~cm}^{-1}$ resolution. The details of those measurements can be found in Ref. [31]. After experimenting with the two demo systems we assembled our prototype from a Headwall Photonics Raman Explorer 785 and an Andor Newton camera as were used in the demo system described above. A different probe and excitation laser wavelength were used for the prototype instrument, hereafter referred to as the Headwall/Andor prototype. Data acquired with this prototype system used an InPhotonics laser Raman probe made for $830 \mathrm{~nm}$ laser excitation, but with a $\mathrm{NA} \approx$ 0.20 . This would also slightly overfill the grating. It utilizes a $100 \mu \mathrm{m}$ diameter excitation core fiber and $200 \mu \mathrm{m}$ collection fiber so will be less efficient than the probe used for the Headwall/Andor demo. Firstly, with the InPhotonics probe the spot diameter on the sample was nearly 2-fold larger than that obtained with the EnWave probe, and secondly, the amount of light passed to each pixel would be reduced about 2 -fold as well, due to throughput mismatch. This would result in more substrate and fluorescence being summed into the signal, and less overall Raman recovered signal, so signal contrast is expected to be less. The InPhotonics, Inc. Raman probe has a depth of focus $>2.5 \mathrm{~mm}$, so field measurements are fairly tolerant of focal position. At the time the prototype was assembled, the InPhotonics probe for $830 \mathrm{~nm}$ was the best commercially available probe for that test wavelength. In partial compensation for its inefficiency to match with the Headwall-Andor spectrograph, we used a more powerful laser source. An Innovative Photonic Solutions spectrum stabilized $830 \mathrm{~nm}$ diode laser system was used. Output power from model no. I0830MU0350MF-TK can be electronically tuned from $300 \mathrm{~mW}$ to $>650 \mathrm{~mW}$ and remains line-locked and has a stable bandwidth to less than $0.08 \mathrm{~nm}$. Power from the InPhotonics Raman probe was measured with a pyrometer and stabilized to $300 \mathrm{~mW}$.

\section{Results}

4.1. Results: Optimal Excitation Wavelength and Spectroscopic Resolution. All things being equal (brightness of illumination source, the NA of the probe-collection optics, magnification, stability and readout noise of the focal plane array, leakage of ambient light, etc.), it is always best to achieve the highest spectral resolution to discern chemical functional groups' signatures. Our tests of oxidants such as sodium chlorate, which have a high elastic Mie-Tyndall scattering cross-section, became a simple test to highlight the limitations of reduced spectral resolution. In Figure 2, the region around the extremely dominant Raman peak was studied as a function of averaging time and also compared to a spectrum obtained under high resolution and extended averaging using a $1064 \mathrm{~nm}$-based FT-Raman system [31].

It is seen that the Headwall/Andor demo system described above is able to much better resolve the peaks in the fingerprint region, whereas with the DeltaNu demo system the peaks are blended. In this comparison, both dispersive systems have integration times quite short compared to the FT-Raman data (4 minutes). The DeltaNu system instrumentally broadens the peaks such that, for example, the minor band at $965 \mathrm{~cm}^{-1}$ is no longer discernible. For the data shown in Figure 2, the main Raman peak located at $935 \mathrm{~cm}^{-1}$ have full-width at half maximum (FWHM) values of $26 \mathrm{~cm}^{-1}$ for the DeltaNu Advantage demo, $9 \mathrm{~cm}^{-1}$ for the Bruker FTRaman, and $10 \mathrm{~cm}^{-1}$ for the Headwall demo system. The estimated optimal FWHM resolution of the DeltaNu Advantage with $1064 \mathrm{~nm}$ excitation is about $14 \mathrm{~cm}^{-1}$ (comparable to that noted in [30]) and that of the Headwall demo system was about $5.5 \mathrm{~cm}^{-1}$, which was somewhat larger than the demo vendor's stated specifications of $4.7 \mathrm{~cm}^{-1}$.

With the Headwall/Andor demo system, the Raman spectra shown in Figure 3 were obtained for liquid filmevaporated coatings of RDX and amino dinitrotoluene. These tests were performed in part to understand the basic sensitivity and resolution tradeoffs of Headwall's novel imaging dispersive Raman spectrograph with a back-illuminated deep-depleted CCD array with a standardized $26 \mu \mathrm{m}$ pixel pitch. The supplied no. 1 slit was stated to be $6 \mathrm{~mm}$ long with a $25 \mu \mathrm{m}$ width.

These first efforts studied thin-films of RDX and amino dinitrotoluene and possible interferents from strongly fluorescing compounds, including skin oils, commonly referred to as sebum. The Raman probes that were used with the Headwall/Andor demo and prototype systems to obtain this baseline data did not make use of optimally high NA collection optics or confocal aperture filtering, so had fairly sizable depth of field values to work with irregularshaped objects with minimal optimization of the samplefocal positions (via manual set up). Mixtures of synthetic sebum were also tested with diluted samples of RDX and other explosives or oxidants. RDX is not as strong a Raman scattering species as sodium chlorate shown in Figure 2, so preliminary tests began with a sequence of pure RDX films and, then its admixture in representative interferent compounds. The data shown in Figure 3 are representative of the background level variations seen with standard samples. Some of the background seen with the dinitrotoluene may be a grey-body emission as well, since this sample had visible color $[13,14]$. The demo tests were made with a representative set of other energetic compounds as well, including smears of commercial Semtex samples on glass slides. The primary design phase effort was to assess the operational limitations to cleanly discern the fastest detonation velocity components, such as RDX and/or PETN in Semtex, above whatever background fluorescence resulted from the matrix of compounds tested. For completeness, we therefore also tested a $1064 \mathrm{~nm}$ spectrograph and optical system from 




(a)
Amino dinitrotoluene compounds $\left(\sim 300 \mu \mathrm{g} / \mathrm{cm}^{2}\right.$ film) on quartz cuvette surface (no background correction)

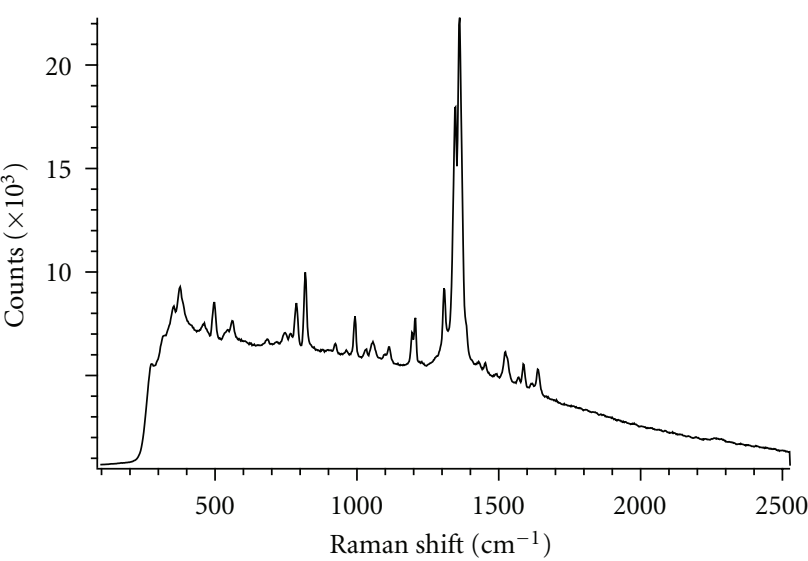

(b)

FIgURE 3: Representative test Raman spectra of pure RDX accreted onto a quartz cuvette (a) and amino dinitrotoluene compound(s) (Supelco) likewise plated to a standard quartz cuvette (b). The spectra were recorded using the Headwall/Andor demo system with $40 \mathrm{~mW}$ of $785 \mathrm{~nm}$ excitation. Each spectrum above is the result of one, $1 \mathrm{~s}$ exposure. The mass, $m$, of material resolved in the focal area of each example is $19.6 \mathrm{ng}$, and $23.6 \mathrm{ng}$ respectively, corresponding to a single rounded-particle radius $r \approx 14.6$ and $15.5 \mu \mathrm{m}$, respectively [via: $m \approx$ $1.5 \mathrm{~g} / \mathrm{cm}^{3} \cdot\left(4 \pi r^{3} / 3\right)$, radius in $\left.\mathrm{cm}\right]$.

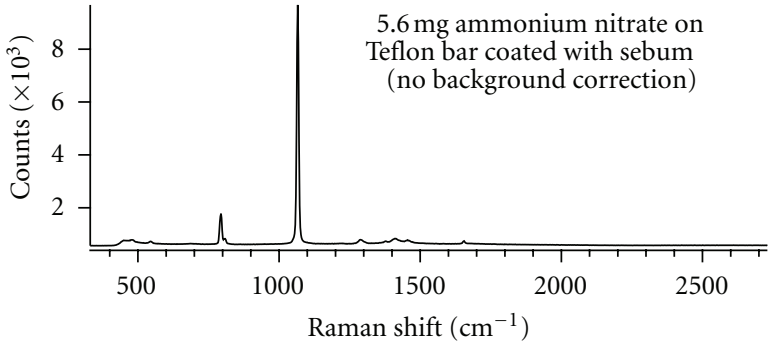

(a)

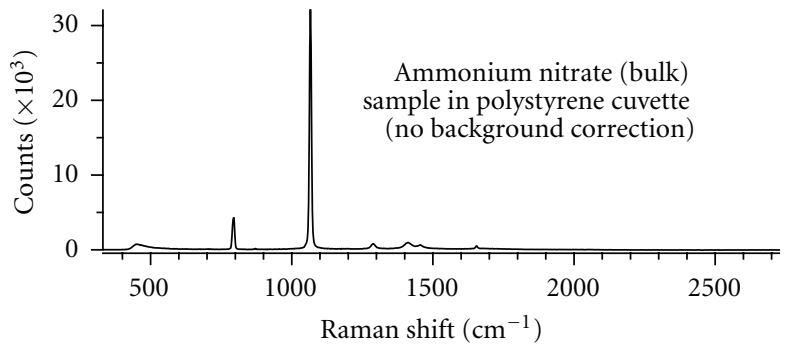

(b)

Figure 4: Raman Stokes spectrum of ammonium nitrate affixed to a Teflon surface with a thick, synthetic sebum coating (a) and loose ammonium nitrate in a plastic cuvette (b). Few effects of contaminants or container wall were observed with strong Raman scattering materials. These spectra were recorded using the Headwall/Andor demo system with $40 \mathrm{~mW}$ of $785 \mathrm{~nm}$ excitation and each is the result of one, $1 \mathrm{~s}$ exposure.

DeltaNu to ascertain whether its design approach would afford substantial improvements of observability of the primary energetic materials by suppressing fluorescence.

In the course of studying neat samples of explosives with the demo systems, a series of spectral interference tests were also performed with fine dustings of solid mixtures of energetic materials and oxidizers placed on or mixed with synthetic sebum coatings added to the substrates. These complex chemical mixtures were deposited upon a variety of metal, glass, and polymer flat surfaces. Figure 4 shows one such intercomparison for the simple case of ammonium nitrate, a relatively strong Raman scatterer. This spectrum was recorded with the Headwall/Andor demo system. In this case, $5.6 \mathrm{mg}$ of ammonium nitrate is affixed to a Teflon bar by a moderate $(\sim 1 / 3$ of a mm thick) layer of synthetic sebum spread over approximately $1 \mathrm{~cm}^{2}$. It is typical of results with a variety of ionic salts, including ammonium sulfate and sodium chlorate. Little effect of spectral interference for these strong ionic scatterers was observable unless the sebum layer was very thick. A spectrum of bulk ammonium nitrate packed into a polystyrene cuvette recorded with the same spectrometer is shown in the lower trace of Figure 4.

\subsection{Results: Wavelength Calibration and System Numerical} Aperture. It was found that the Headwall Photonics Raman Explorer 785 spectrograph needs a fairly dense spread of calibration lines to treat its nonlinear dispersion properties accurately. At the time of this work no reliable Raman spectra (digitized or vetted) for any of the energetic compounds were available. Therefore, in order to validate the $\lambda$ calibration, we studied a variety of ionic salt oxidizers, sulfur particles, polystyrene beads, and so forth. Figure 5 shows 


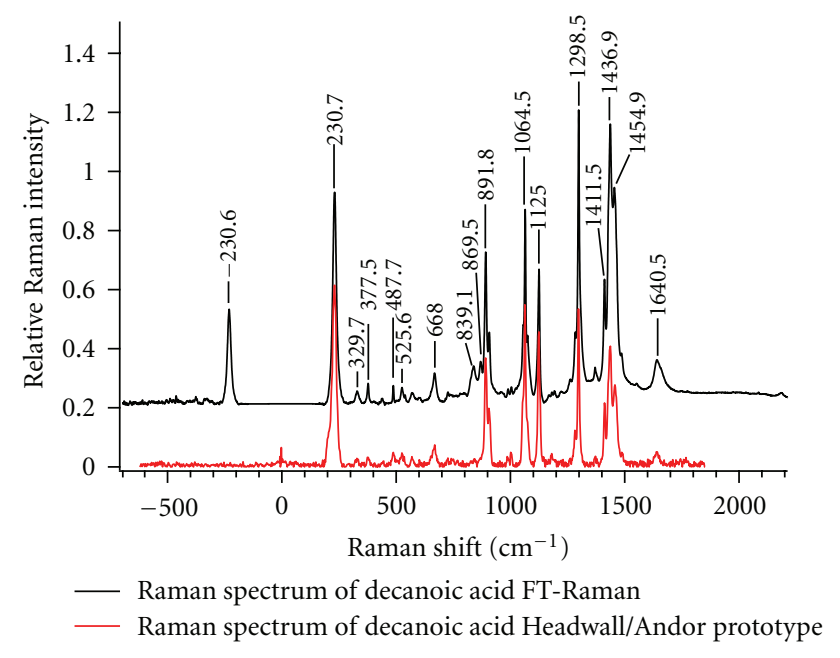

Figure 5: Comparison of Raman signals from decanoic acid. The bottom trace (red) is from the Headwall/Andor prototype dispersive system using $300 \mathrm{~mW}$ of $830 \mathrm{~nm}$ excitation laser for a decanoic acid packed in an aluminum cup. This spectrum is the result of twenty, $1 \mathrm{~s}$ exposures. The top trace (black) is from FTRaman database (at $2 \mathrm{~cm}^{-1}$ resolution) using $300 \mathrm{~mW}$ of $1064 \mathrm{~nm}$ excitation with the sample packed into an aluminum cup. The FTRaman spectrum is the result of coadding 2000 interferograms. The dispersive data are wavelength calibrated against the noted line assignments from the FT-Raman spectrum.

a secondary FT-Raman spectrum of decanoic acid, a fairly stable persistent organic, used to test and then calibrate the Headwall/Andor prototype system. The FT spectrum was taken at $2.0 \mathrm{~cm}^{-1}$ resolution with $1064 \mathrm{~nm}$ excitation [31]. For this calibration work, the FT system had the interferometer frequency calibrated with a stabilized $\mathrm{HeNe}$ laser and the results were compared against the known $1064 \mathrm{~nm}$ excitation laser; that is, calibration appears satisfactory as is seen when comparing the corresponding Stokes/anti-Stokes line shifts. A comparison of the prototype Raman response to the FTRaman data is presented in Figure 5; again the FT-Raman data were acquired with $300 \mathrm{~mW}$ of excitation power, and with averaging times of several minutes compared to a 20 scan summation of $1 \mathrm{~s}$ exposures for the prototype dispersive system.

The comparison of Raman data shown in Figure 5 suggests that the resolution limits for the prototype dispersive spectrograph is fairly satisfactory given that a 25 micron slit is used with a 26 micron pixel spacing. The instrumental lineshape function for this situation would closely approximate a triangle function with $\mathrm{FWHM} \approx 2$ pixels, or about $4.75 \mathrm{~cm}^{-1}$ noting the dispersion factor for Port 1 cited in Section 2. This appears to be nearly satisfied for many lines of interest across the band range of the spectrograph. Surprisingly, relative peak amplitude levels for the prototype dispersive spectrograph are fairly good without any intensity corrections being applied to this raw Raman data, until Stokes shifts exceed about $1300 \mathrm{~cm}^{-1}$ and above. Wavelength calibration appears to be better than 1 pixel, as was verified by comparing the cross-over positions of the first derivative spectra taken from Figure 5.
The acquired decanoic acid FT-spectrum was then used as a wavelength standard to calibrate our dispersive spectrometer. All subsequent results presented were obtained with this Stokes shift data as the secondary calibration standard. In principle, [2] the FT-Raman spectra of persistent "liquid" organics should provide good Stokes shift calibrations in wavenumbers $\left(\mathrm{cm}^{-1}\right)$ anchored to the HeNe line of the interferometer. After calibration of the spectrograph's dispersion, experimental thin films of RDX and TNT were again tested and thin smears of a Semtex sample were also tested as described below.

In the case of thin films of neat explosives evaporated from acetonitrile samples (Supelco) or light physical smears of Semtex, signal counting was done at relatively long update rates. Typically 20 to 100 of the $1 \mathrm{~s}$ integrated scans were acquired to see good evidence of the thinnest films studied. A noticeable large background signal of complex shape was observed at these low-concentration sample conditions. Careful shielding of the spectrograph and probe head from room lights suggested it was not a light leakage effect. The background had no apparent origin from the coupons themselves but appears to be an artifact in the spectrographCCD camera system. Other than for its integrated amplitude, the shape of the background was nearly the same whether we used glass slides or metal coupons. It had a fairly consistent spectroscopic profile at low Raman signal conditions, so could be readily subtracted through a baseline subtraction routine.

There appeared to be little need to apply the baseline correction to strong Raman signals from bulk samples or single grains of ionic salts, however. This leads us to speculate that the effect of the baseline is a residual defect of the CCD readout array or photochromic stain on the CCD's entrance window. To test this hypothesis, the data of Figure 6 show the uncorrected Raman spectra for both a bulk sample of neat sulfur as well as small single sulfur particle manipulated into the Raman probe's focal spot of maximum response. These data illustrate that the low signal level nonlinear baseline is neither a problem associated with strong scattering samples nor when a single particle is interrogated in the focus of the Raman probe with its large numerical aperture, but rather arises from a readout defect of the CCD array as posited above.

The depth of field (DoF) and nominal $100 \mu \mathrm{m}$ diameter spot size of the $830 \mathrm{~nm}$ Raman probe of the Headwall/Andor prototype system allowed decent signal capture with simple manipulations of small grain particles by eye and slight manual manipulation of simple kinematic stages without need for microscopic visualizations or vernier controls. Neat sample Raman spectra or spectra obtained from distinguishable grains or specks of samples were all analyzed by simple manipulation of slides. Rapid updates allowed fine manual tuning of particulates and/or simple $X-Y$ translations.

\subsection{Results: Experimental Prototype System Crafted to Study} Semtex Components. When studying the Raman spectra of various energetics, the work of Lewis et al. $[18,22]$ showed some of the wavelength-dependent nuances of the Semtex 


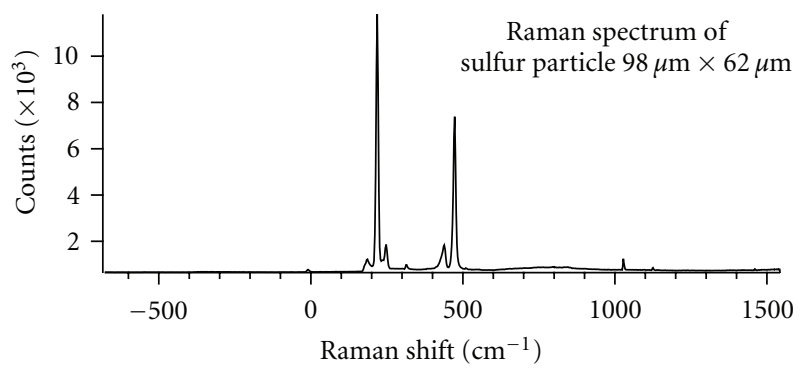

(a)

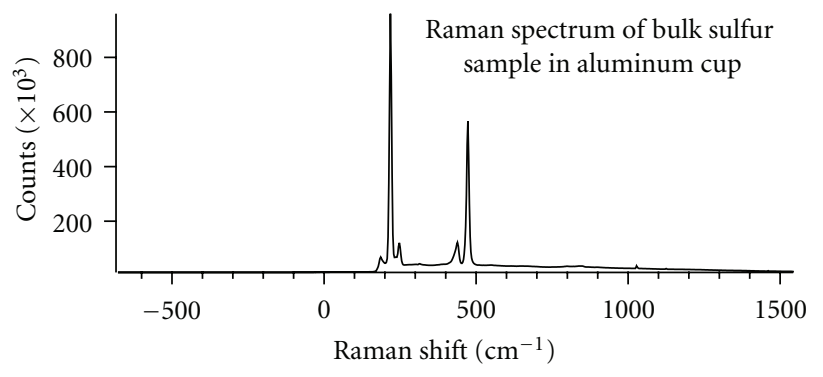

(b)

Figure 6: Raman Stokes spectra (uncorrected baseline) of a single sulfur particle (a) interrogated in the focus of the Raman probe on a metal coupon compared to a neat packed sample of sulfur (b). These spectra were recorded using the Headwall/Andor prototype spectrometer, $6 \mathrm{~mm} \times 25 \mu \mathrm{m}$ slit, 200 lines $/ \mathrm{mm}$. Both spectra were recorded with $300 \mathrm{~mW}$ of $830 \mathrm{~nm}$ laser light. The particle spectrum was recorded with a single $1 \mathrm{~s}$ exposure. The bulk sample spectrum was recorded with twenty, $0.2 \mathrm{~s}$ exposures.

system, and how its Raman spectra were more complex than other explosives. We have therefore extended the evaluation of Headwall/Andor prototype system to this compound in particular. Figures 7-9 illustrate the baseline corrected Raman Stokes spectra for deposited films of a Semtex smear of unknown provenance and neat RDX, a primary component of Semtex. The RDX was evaporated from a supplied 1,000 ppm (by weight in $1 \mathrm{~mL}$ of acetonitrile) standard stock solutions (Supelco) onto metal coupons. In this case, the RDX was on an aluminum coupon. Sample substrate was a case by case matter: some were on aluminum, others on steel. The metal coupons showed no intensity changes, nor did there appear to be any changes to the Stokes shifts. The Semtex sample was supplied sandwiched between a pair of glass slides and carefully separated for testing without any cover. Note that a very similar baseline correction was applied to each case, despite using very different substrates in these comparisons.

After calibration of the Raman spectra with our decanoic acid standard, the peak positions were calculated using the Bruker software OPUS "standard" peak picking utility which uses a first derivative and interpolation algorithm to find the peak centers. The thin-film Semtex sample with the background spectrum shown in Figure 7-was provided to us with no information as to its origin, composition, or concentration. A thicker portion of this Semtex sample was also studied and produced a somewhat different Raman

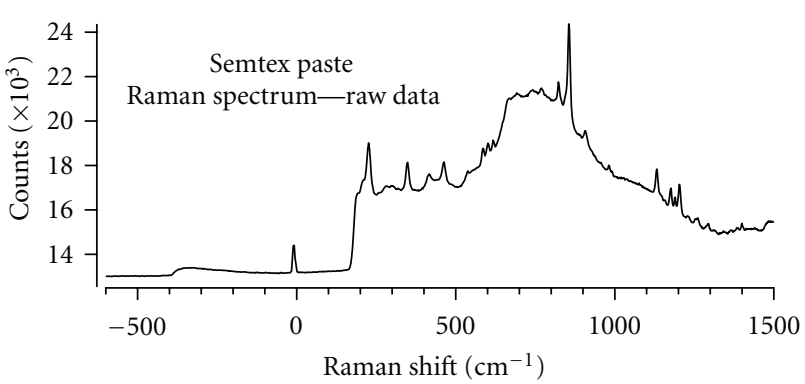

(a)

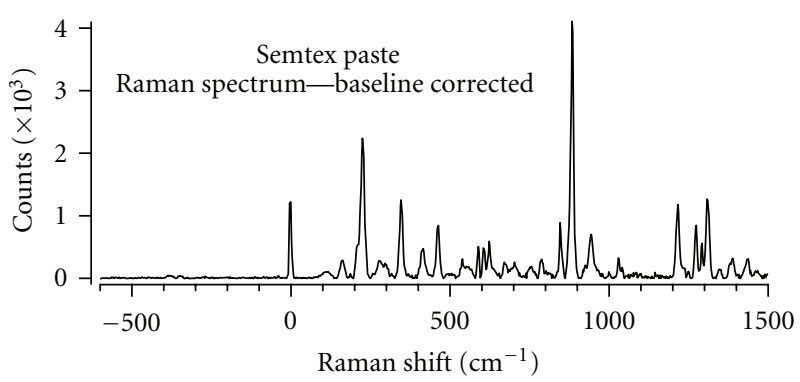

(b)

Figure 7: Example of wavelength calibrated thin-film study of Semtex sample on a glass microscope slide using the Headwall/Andor prototype system, $6 \mathrm{~mm} \times 25 \mu \mathrm{m}$ slit, 200 lines $/ \mathrm{mm}$. The spectrum was recorded using $300 \mathrm{~mW}$ of $830 \mathrm{~nm}$ laser light. The Andor Newton CCD camera accumulated twenty, $1 \mathrm{~s}$ exposures. The wavenumber scale was calibrated using the decanoic acid spectrum shown in Figure 5. The upper trace is raw data, and the lower trace is baseline corrected utilizing a simple spline fit algorithm in Bruker's OPUS software. The large offset between the Rayleigh peak $\left(0 \mathrm{~cm}^{-1}\right)$ and leftmost column of pixels (with extrapolated Raman shift of $\sim-630 \mathrm{~cm}^{-1}$ ) could potentially be exploited with Vnotch-filter-based Raman probes to view the dominant anti-Stokes lines from lower order lattice vibrations. The edge filter of the Headwall/Andor prototype probe system does not allow us to access this low wavenumber region.

spectrum as shown in Figure 8. The spectra in Figures 7 and 8 were obtained using the prototype $830 \mathrm{~nm}$ based Raman chemical analyzer. Figure 8 below shows the quality of spectra obtainable with paste smears on glass surfaces collecting 20 scans of $1 \mathrm{~s}$ averaged data. For comparison, the FT-Raman data $\left(2 \mathrm{~cm}^{-1}\right.$ resolution) were obtained with an excitation laser power of $25 \mathrm{~mW}$ and 4 minute average using a bulk commercial Semtex-H sample in a metal cup. With the exception of an odd and broad spectral peak near $700 \mathrm{~cm}^{-1}$, and minor variations of the crystalline peaks associated with RDX at 400 to $500 \mathrm{~cm}^{-1}$, the dispersive spectrum of Figure 8 is fairly consistent with the intensity corrected FT-Raman spectrum of Semtex-H. At Stokes shifts $\gtrsim 1300 \mathrm{~cm}^{-1}$, the spectrum from the dispersive system starts to drop, presumably due to the lower sensitivity of the SiCCD camera response for wavelengths $>950 \mathrm{~nm}$.

The ultimate objective of a good field Raman analyzer would be the assignment of the mixtures of RDX and PETN used to formulate various Semtex compositions. Towards this end, we studied RDX under thin film conditions, to 


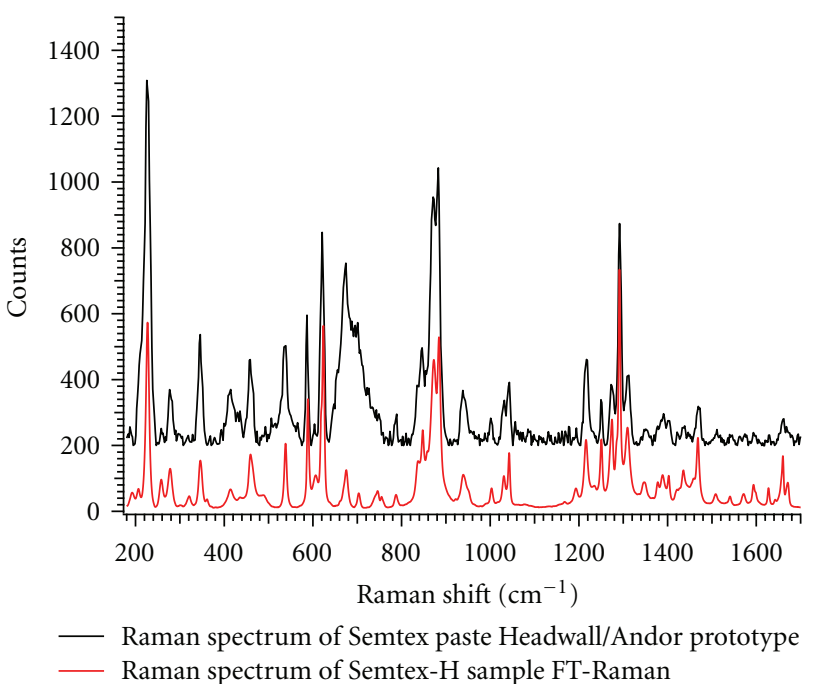

Figure 8: Comparison of prototype dispersive Raman signal of unknown Semtex paste sample (top trace) against a calibrated and intensity corrected FT-Raman spectrum from commercial Semtex$\mathrm{H}$ bulk sample (lower trace).

understand possible changes in phase structure. In addition to studying evaporated thin films of RDX, we also studied discrete particles of RDX and many other materials. The particles were formed by scraping thin films of the materials on glass slides with a razor blade and then using a machinist's microscope with a calibrated reticle to size the particles. Generally, the detection sensitivities for these two sample types compared well for net mass of sample to see similar signal strength. The particle masses were determined by using the machinist's microscope to measure the size of the particles and then using the known density of the materials to estimate the particle mass. In the case of the RDX studies, the particles were fairly rounded (so assumed spherical) and their mass, $m$, was estimated as $m \approx\left(1.5 \mathrm{~g} / \mathrm{cm}^{3}\right) \cdot\left(4 \pi r^{3} / 3\right)$. In the Raman Stokes data shown in Figure 9, the derived or equivalent areal dosage of the RDX particulate was about $1.1 \mathrm{mg} / \mathrm{cm}^{2}$.

Table 2 provides a list of the prominent lines found in Figure 9 compared against commonly accepted literature results for the RDX Raman Stokes spectral peaks [6]. Other than a few low frequency bands, the positions of most of the peaks are within the stated FWHM resolution of the Headwall spectrograph $\left(4.7 \mathrm{~cm}^{-1}\right.$ with $26 \mu \mathrm{m}$ pixel pitch), indicating good wavelength calibration across the spectrum.

Figure 10 shows expanded FT-Raman spectra for RDX and PETN in the fingerprint region from the FT-Raman system [31]. Figure 11 shows Raman Stokes spectra from the FT-Raman database for a sample of Semtex-H which can have a nearly $1: 1$ ratio of RDX to PETN, although this is variable with up to a maximum of $65.5 \% \mathrm{RDX}$ and a minimum of $25 \%$ PETN according to Moore et al. [32]. From visual inspection of these spectra, it is straightforward to see the origins of certain lines in the Semtex-H Raman data shown in Figure 11, which in this case would appear to have about $\sim 1: 1$ mix of each active compound. The RDX is
TABLE 2: Comparison between peak picks of $830 \mathrm{~nm}$ laser excitation of Raman Stokes lines and reference RDX Raman $\left(\mathrm{cm}^{-1}\right)$ peak positions using $632.8 \mathrm{~nm}$ excitation and $1064 \mathrm{~nm}$ excitation.

\begin{tabular}{lcc}
\hline $\begin{array}{l}830 \mathrm{~nm} \text { data } \\
\left(\mathrm{cm}^{-1}\right)\end{array}$ & $\begin{array}{c}\text { Reference [6] } \\
\left(\mathrm{cm}^{-1}\right)\end{array}$ & $\begin{array}{c}\text { Reference }[31] \\
\left(\mathrm{cm}^{-1}\right)\end{array}$ \\
\hline 158 & 152 & 147 \\
224 & 226 & 225 \\
345 & 345 & 346 \\
412 & 413 & 414 \\
460 & 463 & 463 \\
590 & 589 & 590 \\
605 & 606 & 606 \\
667 & 668 & 669 \\
788 & 787 & 787 \\
846 & 849 & 848 \\
884 & 885 & 884 \\
944 & 944 & 944 \\
1028 & 1031 & 1030 \\
1216 & 1217 & 1216 \\
1272 & 1272 & 1273 \\
1309 & 1312 & 1310 \\
1346 & 1350 & 1346 \\
1389 & 1390 & 1388 \\
1434 & 1426 & 1435 \\
1571 & 1573 & 1572 \\
1595 & 1595 & 1593 \\
\hline
\end{tabular}

assumed to be mostly in the $\alpha$-RDX phase, but there is some variance seen in the broader spectral features between 400 to $\sim 500 \mathrm{~cm}^{-1}$ that could be representative of a small mixture with $\beta$-RDX [33-35]. The lower trace of Figure 11 shows a simple additive simulation of the Semtex-H assuming PETN and RDX in equal proportion and not using any other linear superpositions for the matrix of binder-plasticizers. This work is almost a "textbook" example of how intensity corrected Raman line maps can often be used to do additive or subtractive analyses of a mixed material's composition. Akhavan was the first to demonstrate this capability with an FT-Raman system using $1064 \mathrm{~nm}$ excitation for the three common variants of Semtex that are commercially available [16]. Effects of binders and plasticizers that would account for about 13 to $14 \%$ of the material weight seem slight in this spectral band.

In this same simple fashion, we studied a thin-film Semtex sample of unknown provenance (see Figure 7). We first assume that the baseline correction is not adversely rejecting a larger diffuse background signal from the binder and plasticizer stock, which seems to be a fair assumption based on the simulation above. However, fluorescence and scatter could be larger in the case of $830 \mathrm{~nm}$ excitation. Figure 12 (lower trace) shows the baseline corrected Raman spectrum of this thin-film Semtex sample recorded using the Headwall/Andor prototype system, and this is compared to the simulated spectrum (upper trace) that assumes a RDX: PETN mixture in the ratio of $7: 2$ to largely match (i.e., 


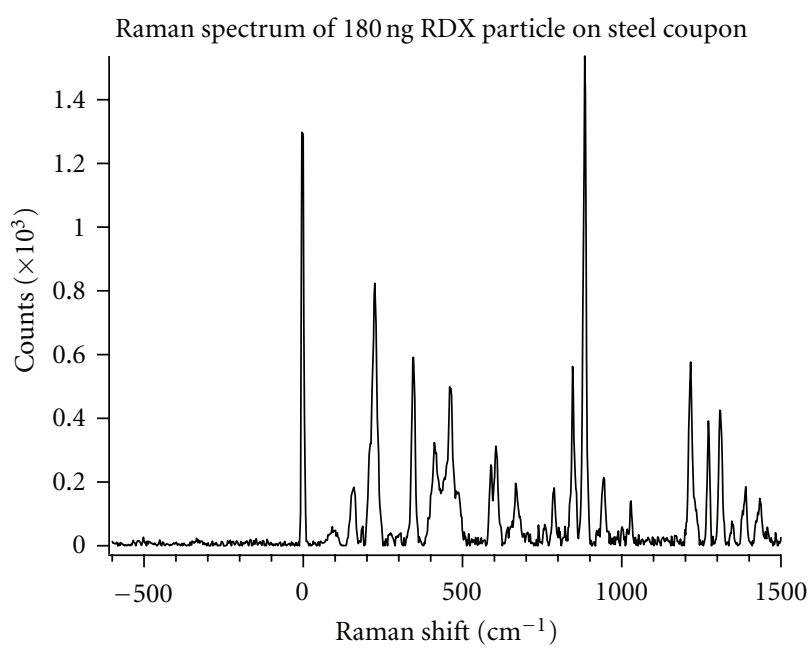

FIGURE 9: Raman Stokes signal of a $180 \mathrm{ng}(\sim 60 \mu \mathrm{m}$ diameter $)$ RDX particle on a steel coupon. The spectrum was recorded using the Headwall/Andor prototype system, $6 \mathrm{~mm} \times 25 \mu \mathrm{m}$ slit, 200 lines $/ \mathrm{mm}$. The particle was illuminated with $300 \mathrm{~mW}$ of $830 \mathrm{~nm}$ laser light. The Andor Newton CCD camera accumulated twenty, $1 \mathrm{~s}$ exposures. The wavenumber scale was calibrated using the decanoic acid spectrum shown in Figure 5. The spectrum was baseline corrected utilizing a simple spline fit algorithm in Bruker's OPUS software.

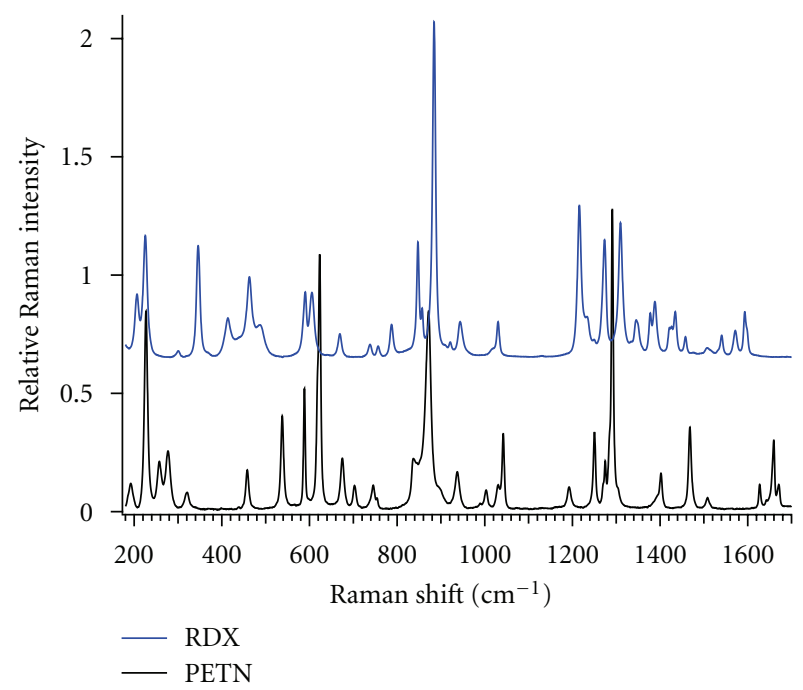

FIGURE 10: Intensity corrected and normalized FT-Raman spectra of pure RDX (offset in upper trace) and PETN (lower trace) excited with a $1064 \mathrm{~nm}$ laser. Instrument resolution was set to $2 \mathrm{~cm}^{-1}$. Both spectra were obtained with a Bruker FT-Raman system using the protocols described in [31]. The FT-Raman spectra of the pure materials were scaled from 0 to 2.0 for the largest band.

manually adjusted the ratio in a computer simulation) the multiple peak structure seen between 1200 and $1350 \mathrm{~cm}^{-1}$. That spectral region has a smooth baseline correction and looks fairly clean of matrix effects and any polymorphisms of the RDX based on the simulation above. Another good region might be the triplet of lines near $600 \mathrm{~cm}^{-1}$, but in the $830 \mathrm{~nm}$ Headwall system this is near a region of poor baseline correction.

\section{Discussion as to System Performance and Needed System Improvements}

Preliminary studies of a high-resolution Offner-Dyson dispersive spectrograph (Headwall Raman Explorer 785) indicates it is a solid, wavelength stable "spectral engine" and potentially suitable for fast line imaging or multitrack fiber imaging of explosives using modern CCD arrays. The current embodiment was tested with a $26 \mu \mathrm{m}$ pixel pitch CCD array (Andor-Newton with back-illuminated deep-depleted array option), which is close to the recommended $25 \mu \mathrm{m}$ pitch needed to achieve an average reciprocal linear dispersion of $2.37 \mathrm{~cm}^{-1}$ per pixel using Port 1 . While this can resolve a peak spectrally centered exactly on pixel with a Nyquist limit to resolve the base of symmetric lines with 2 pixels, it is better to oversample if baseline scatter and other matters of irregular transmission or vernier étalon structure are to be better accounted for. A preferable combination would be a $25 \mu \mathrm{m}$ input slit coupled with a $13 \mu \mathrm{m}$ pixel pitch CCD array with similar spectroscopy grade quantum efficiency into the deep near-IR.

Wavenumber calibration of the Headwall/Andor prototype system was easily achieved by recording the Raman spectrum of a stable organic compound with sufficient line density to account for the nonlinearity of the spectrograph's output. The use of $830 \mathrm{~nm}$ light for excitation was also shown to be a reasonable compromise wavelength between signal strength and background fluorescence. Headwall Photonics now manufactures a Raman Explorer model optimized for use with $830 \mathrm{~nm}$ light. The linearity of the prototype system's intensity response was demonstrated by its ability to disentangle the composition of the Semtex sample (see Figure 12). The prototype's sensitivity was demonstrated by the spectrum of the $60 \mu \mathrm{m}$ diameter (180 ng) particle of RDX shown in Figure 9. While the signal to noise for this size particle is good for "off-the-shelf" components, further improvements will be needed, particularly in the coupling of the probe to the spectrograph and camera performance, to achieve detectivity for particles $10 \mu \mathrm{m}$ or less in diameter.

Newer CCDs with excellent and nearly flat quantum efficiency in the deeper near-IR close to Si-band-gap are feasible for use with this standardized spectrograph based on research by Oluseyi et al. using "Hi-Rho" silicon [36]. To our knowledge, these high-resistivity Si-CCD arrays are still not presently available from the licensed vendor of that technology, but a recent article describes commercial testing of high resistivity CCDs [37]. If such arrays become available, they would improve detection of the Raman Stokes lines from $830 \mathrm{~nm}$ illumination by nearly $40 \%$ and could allow more uniform intensity corrected performance over the practical Stokes line shifts out to $1730 \mathrm{~cm}^{-1}$. The bands near the nominal $1700 \mathrm{~cm}^{-1}$ Stokes shifted range encompass some important lines that can distinguish and quantify PETN and RDX in difficult to study "cocktail mixes" of Semtex or other plastic explosive mixes that may emerge. 


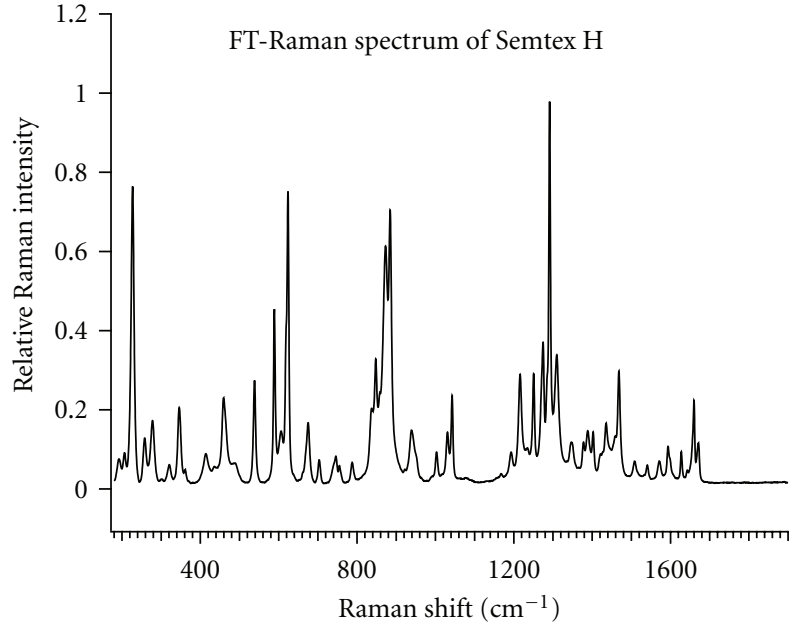

(a)

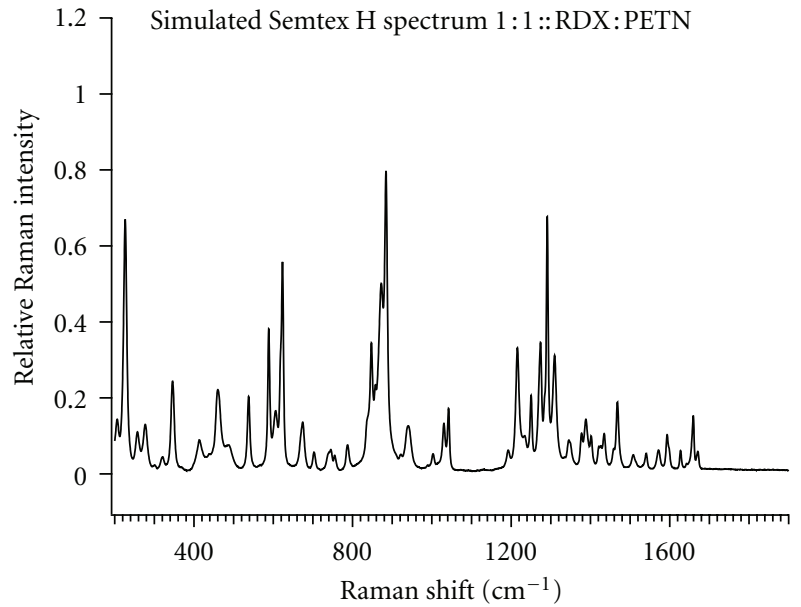

(b)

Figure 11: Database Raman spectrum of a commercial Semtex-H sample using a Bruker 1064 nm FT-Raman spectrometer (a) and a simulated Semtex-H Raman spectrum assuming a 1:1 mix of RDX and PETN without binder(s) or plasticizer(s) contributions (b). For the $1064 \mathrm{~nm}$ data, the spectra were scaled from 0 to 2.0 for the largest band.

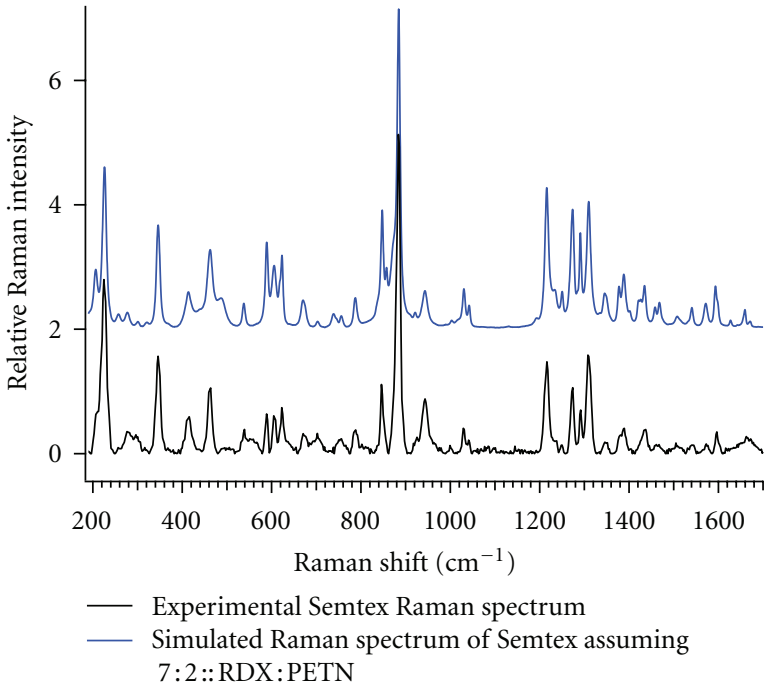

Figure 12: The lower trace of this figure shows the experimental Raman spectrum of the thin-film Semtex sample of unknown provenance taken with the $830 \mathrm{~nm}$ prototype Raman imager. This is an expanded view of the spectrum shown in Figure 7, lower trace. The upper trace is a composite sum of the FT-Raman spectra of PETN and RDX Raman Stokes signals taken from Figure 10 (with an offset baseline). These spectra were used to derive the additive spectrum with RDX and PETN in a $7: 2$ ratio.

Another needed improvement to make line epi-illumination and spectral line-imaging practical is the Raman probe or fore-optics. Several researchers have extensively researched the advantages of line illumination $[12,25,26]$, yet surprisingly, no commercial variant of this idea has emerged. McCreery has also shown that the chemical analysis of heterogeneous samples can be greatly improved for applications such as Raman analysis of pills, by rapidly summing the multi-track spectra from a spinning target [12]. To date, most spatially multiplexed solutions of fast Raman sample analysis use stacked or bundled multifiber probes, and these may have alternate benefits to improve the illumination conditions that permit greater depth of field tolerance per Schrum et al. [38]. However, based on McCreery's work $[12,29]$, probes with Powell lens illuminators [39] or other derivative variants would provide a substantial improvement in sensitivity, quite possibly pushing quantitative sensitivity from the $\sim 300 \mu \mathrm{g} / \mathrm{cm}^{2}$ areal dose levels seen in Figure 3 to the few or less than $\sim 1 \mu \mathrm{g} / \mathrm{cm}^{2}$ dosages desired for rapid field analyses. While confocal Raman microscopy can quantify picogram samples, these techniques are not suitable for harsh field operations and are still labor intensive as an operator must manually sequence interesting flecks of substance.

\section{Design Considerations for a Line-Imaging Spectrometer}

A final objective of our work is to evaluate the design criteria for a robust line-illuminating, line-imaging Raman spectrograph based on the recent techniques of making optically fast (low F/\#) stigmatic imaging grating spectrographs. Figure 13 shows the basic architecture we are striving to devise based on an Offner-Dyson spectrograph [11]. It would be a line illumination variant of the confocal spectral imaging (CSI) systems that Renishaw first devised [6] but uses an OffnerDyson spectrograph as the dispersive engine to enhance pixel spectral sampling resolution interval to about 2.37 or $1.64 \mathrm{~cm}^{-1}$ per pixel assuming a $26 \mu \mathrm{m}$ array spacing. The operational objective would be to simplify the input optics and detect direct backscattering with the same focusing optic (epi-illumination) and use a line source of laser light to reduce the specific intensity of focused excitation light on a target, but be able to integrate signals with overall greater 




Figure 13: An example line formed epi-illuminator and line image transfer optic to match the target line illumination to a low distortion Offner-Dyson spectrograph (slit intensity shown in the inset). The 1st cylindrical achromat reshaping light from a $1 \mathrm{D}$ axicon (Powell lens) is oriented at 90 degrees with respect to the focusing objective (2nd) and transfer (3rd) cylindrical achromats. The line image was designed in this case to be $12 \mathrm{~mm}$ high. The illuminated/imaged sample is shown in the lower left of the figure.

average total power. Following McCreery's suggestions of using line imaging with rotating samples to enhance sample averaging [12], it is expected that the system would be a dual use portable chemical imaging system that could look at swipe smears of compounds on irregular surfaces or perhaps fingerprints transferred to circular metal coupons. Thus the system would be designed to be field hardened, serving as a mobile precision laboratory system, but capable of doing in situ studies of fixed stains. Headwall has devised a number of Offner-designed industrial grade spectrographs for both hyperspectral imaging in the near-IR, and for Raman line imaging applications [28]. These designs are highly corrected for astigmatism, spherical aberration, coma, and field curvature. Astigmatism is the strongest aberration to reduce spatial signal summing and scales quadratically with both the NA of the spectrograph and with the angle of off-axis rays from the centerline of the spectrograph [26]. The Headwall design reduces the distortions, known as smile and keystone, resulting from astigmatism and other aberrations to $<0.1 \%$. While it may not be as ideal for trace residue analysis as the multichannel FT-Raman spectrometer devised by Zhao and McCreery [29], it should serve well as a multitrack imager or line imaging Raman spectrograph. At this preliminary design juncture, we have verified wavelength calibration but have not accounted for the relative intensity response of the CCD device.

The first notable dispersive Raman imager that had low smile and keystone distortion properties for making clean Raman line images was the Kaiser Optical Systems, Inc., (KOSI) holographically corrected transmissive spectrograph $[23,24]$. This system has been thoroughly evaluated at Griffiths' lab in the 1990s [18-22] and found to be effective at studying mixed explosives with low overall response to fluorescence from fuel oils, binders, stabilizers, and taggants added to compounds such as Semtex [22]. The work by Lewis et al. utilized a variant of the KOSI spectrograph to assess the ability to suppress fluorescence from nonexplosive additives while providing high resolution Raman spectra of the energetic constituents of Semtex and other mixed compounds. That work showed that laser excitation at $785 \mathrm{~nm}$ is effective with most mixed formulations, but that with variants of Semtex, it was more difficult to differentiate its primary explosive constituents due to presence of strong fluorophores. The Lewis' were the first to recommend that slightly longer laser excitation at 840 to $860 \mathrm{~nm}$ would be a good compromise of cost to resolve the two explosives, pentaerythritol tetranitrate (PETN) and RDX in Semtex, while mostly suppressing the fluorescence of its various polymeric binders, plasticizers, stabilizers, and colorants [18, 22].

Figure 13 shows an example of our recent ray-tracing results to use commercially available achromatic cylindrical lenses to create a long, sharp focused line-width of epiillumination on target, with the feature of transferring the backscattered Raman signal onto the Headwall slit with nearly matched NA and little magnification penalty. It should yield nearly diffraction-limited line imaging and, if desired, higher magnification could be introduced for such work. Based on ray-tracing of this concept and several of its variants, a cylindrical lens probe would have the potential for little defocus at the target down to $\sim 5$ to 8 microns at $830 \mathrm{~nm}$. The design shown has a practical small parts count and flexibility to vary the focal lengths to better match field conditions. It is currently designed around F/2 commercial optics, which could give a theoretical line image of $2.44 \cdot \lambda \cdot \mathrm{F} / \#$, or about $\approx 4 \mu \mathrm{m}$.

\section{Conclusions}

Fast, noncontact field analysis of complex chemical stains or particles spread over surfaces of several square centimeters is the final goal of our work. The path for quantifying the chemical makeup of smaller particles $<10 \mu \mathrm{m}$ or $1.5 \mathrm{~g} / \mathrm{cm}^{3} \cdot 4 \pi / 3 \cdot\left\{r^{3}\right\} \leq 0.785 \mathrm{ng}$ or finely suspended thinfilms at areal-dosages of $\leq 1 \mu \mathrm{g} / \mathrm{cm}^{2}$, while not fully achieved in this study, does look feasible. Sensitive quantification of trace explosive stains or barely visible particles, without the need to use confocal Raman spectroscopy, appears practical with our prototype system. Emerging optical technologies that would offer greater throughput (étendue) advantages and quantum efficiencies should greatly improve the overall performance. In this work, we also undertook to begin quantifying thin film swipes of Semtex with the goal of establishing a baseline of performance and, to understand if the origin of the samples can be established based on (preferably additive) superpositions of quantitative Raman spectra. It was observed that thin films do not give excellent ratios of the RDX and PETN ratio that thicker paste-like coatings can provide. This matter of heterogenous differentiation of the components will need more study if there is need to quantify the relative mixture of cocktail mixes of Semtex. We have shown that a reliable FT-Raman database that was intensity corrected and carefully normalized for sample concentrations looks promising to subtract or correlate the 
constituents of mixed explosives. The database is growing and will include many compounds needed in disentangling the constituents of mixed explosives, and we have seen some good preliminary matches of composite data with lab based studies of thicker smears. Based on our preliminary results, spectral reconstructions will be informative if we can achieve Raman Stokes resolution somewhat better than a CCD array Nyquist-limited $\sim 4 \mathrm{~cm}^{-1}$.

Requisite spectral resolution to get oversampled spectra with a $4 \mathrm{~cm}^{-1}$ resolution (FWHM) using an existing, nomoving-parts, dispersive Offner-Dyson spectrograph is feasible with arrays having 12 to $15 \mu \mathrm{m}$ pixel pitch transverse to the slit direction. The preferred array should be a high resistivity $\mathrm{Si}-\mathrm{CCD}$ array with high quantum efficiency through to at least $\approx 970 \mathrm{~nm}$. This is now possible with high resistivity $\mathrm{Si}$-CCDs that may greatly improve overall intensity uniformity to the deeper NIR.

The next phase of research will focus on devising robust line imagers that are throughput matched to the spectrograph. We illustrated one such variant using new Powell lens optics (1D axicon) and three cylindrical achromat lenses to form a tightly focused, but a long line $(12 \mathrm{~mm})$ of illumination that can be tailored in length using a simple fiber output lens. The resulting backscattered light from the epi-illuminated target can be transferred with low distortion and throughput matched to an 8 to $15 \mu \mathrm{m}$ wide slit. This would give a Nyquist resolution better than $4 \mathrm{~cm}^{-1}$ in a fairly compact system that has been industrially hardened against dust, weather, and vibrations. The significant limitation to this spectral engine's effective application in field work would be the CCD array's low-signal cooling demands and readout noise limits.

In order to reduce cooling needs, methods of wavelength modulation will be reviewed in order to better correct array read-out nonlinearities and offsets. Methods of using a tunable near-IR excitation to help achieve large background suppression look quite promising, as are advanced methods of ex post facto data treatments, reviewed by Moore and Scharff [40]. In our own early work on hyperspectral line imaging with near-IR and short-wave IR mechanically scanned spectrographs, simple wavelength tuning of just 1 to 2 pixel's range of wavelength can significantly reduce array readout noise and background fluctuations through subtractive techniques. The resulting signals are derivative in shape, but can be reverted to a very close approximation of the original lineshapes by numerical integration. SNR improvements of more than 10-fold have been seen with this concept to suppress common-mode detector array gain offsets, and there may be effective methods to do wavelength modulation reliably with the fixed grating Offner spectrographs that do not add much penalty to cost or ruggedness.

\section{Acknowledgments}

The research described in this paper was supported by the Combating Terrorism Technical Support Office (CTTSO) and the Joint Improvised Explosive Device Defeat Organization (JIEDDO). The authors thank their sponsors for support. The Pacific Northwest National Laboratory is a multiprogram national laboratory operated by Battelle for the U.S. Department of Energy under Contract DEAC05-76RL01830. A portion of the research was performed using EMSL, a national scientific user facility sponsored by the Department of Energy's Office of Biological and Environmental Research and located at Pacific Northwest National Laboratory.

\section{References}

[1] J. M. Chalmer, H. G. M. Edwards, and M. D. Hargreaves, Eds., Infrared and Raman Spectroscopy, John Wiley \& Sons, Chichester, UK, 2011, See Sec. III; Counter Terrorism and Homeland Security.

[2] S. D. Harvey, M. E. Vucelick, R. N. Lee, and B. W. Wright, "Blind field test evaluation of Raman spectroscopy as a forensic tool," Forensic Science International, vol. 125, no. 1, pp. 12$21,2002$.

[3] D. S. Moore and K.-Y. Lee, "Raman spectroscopy as a tool for long-term energetic material stability studies," Journal of Raman Spectroscopy, vol. 38, no. 9, pp. 1221-1224, 2007.

[4] M. L. Lewis, I. R. Lewis, and P. R. Griffiths, "Raman spectroscopic studies of explosives and precursors: applications and instrumentation," in Infrared and Raman Spectroscopy, 5.2, John Wiley \& Sons, Chichester, UK, 2011.

[5] M. D. Hargreaves, R. L. Green, W. Jalenak, C. D. Brown, and C. Gardner, "Handheld Raman and FT-IR spectrometers," in Infrared and Raman Spectroscopy, 5.3, John Wiley \& Sons, Chichester, UK, 2011.

[6] C. Cheng, T. E. Kirkbride, D. N. Batchelder, R. J. Lacey, and T. G. Sheldon, "In situ detection and identification of trace explosives by Raman microscopy," Journal of Forensic Sciences, vol. 40, no. 1, pp. 31-37, 1995.

[7] A. G. Mercado, J. Janni, and B. Gilbert, "Image analysis of explosives fingerprint contamination using a Raman imaging spectrometer," in Law Enforcement Technologies: Identification Technologies and Traffic Safety, vol. 2511 of Proceedings of SPIE, pp. 142-152, June 1995.

[8] J. R. Verkouteren, "Particle characteristics of trace high explosives: RDX and PETN," Journal of Forensic Sciences, vol. 52, no. 2, pp. 335-340, 2007.

[9] J. MacDonald, "Optical microscopy," in Microelectronics Failure Analysis: Desk Reference, R. J. Ross, Ed., p. 472, ASM International, Materials Park, Ohio, USA, 6th edition, 2011.

[10] R. C. Kain, "Micro-Imaging System," US Patent No. 5, 754, 291, May 19, 1998.

[11] D. R. Lobb, "Theory of concentric designs for grating spectrometers," Applied Optics, vol. 33, no. 13, pp. 2648-2658, 1994.

[12] R. L. McCreery, "Raman spectroscopy for chemical analysis," in Chemical Analysis, vol. 157, p. 114, Wiley-Intersciences, John Wiley \& Sons, New York, NY, USA, 2000.

[13] K. L. McNesby, J. E. Wolfe, J. B. Morris, and R. A. PesceRodriquez, "FT-Raman spectroscopy of some energetic materials and propellant formulations," Army Research Laboratory Report ARL-TR-233, 1993.

[14] N. F. Fell, J. M. Widder, S. V. Medlin, J. B. Morris, R. A. PesceRodriguez, and K. L. McNesby, "Fourier transform Raman spectroscopy of some energetic materials and propellant formulations," Journal of Raman Spectroscopy, vol. 27, no. 2, pp. 97-104, 1996. 
[15] S. D. Williams, T. J. Johnson, T. P. Gibbons, and C. L. Kitchens, "Relative Raman intensities in $\mathrm{C}_{6} \mathrm{H}_{6}, \mathrm{C}_{6} \mathrm{D}_{6}$, and $\mathrm{C}_{6} \mathrm{~F}_{6}$ : a comparison of different computational methods," Theoretical Chemistry Accounts, vol. 117, no. 2, pp. 283-290, 2007.

[16] J. Akhavan, "Analysis of high-explosive samples by Fourier transform Raman spectroscopy," Spectrochimica Acta A, vol. 47, no. 9-10, pp. 1247-1250, 1991.

[17] C. M. Hodges and J. Akhavan, "The use of Fourier transform Raman spectroscopy in the forensic identification of illicit drugs and explosives," Spectrochimica Acta A, vol. 46, no. 2, pp. 303-307, 1990.

[18] I. R. Lewis, N. W. Daniel Jr., N. C. Chaffin, P. R. Griffiths, and M. W. Tungol, "Raman spectroscopic studies of explosive materials: towards a fieldable explosives detector," Spectrochimica Acta A, vol. 51, no. 12, pp. 1985-2000, 1995.

[19] I. R. Lewis, N. W. Daniel, and P. R. Griffiths, "Interpretation of Raman spectra of nitro-containing explosive materials. Part I: group frequency and structural class membership," Applied Spectroscopy, vol. 51, no. 12, pp. 1854-1867, 1997.

[20] M. L. Lewis, I. R. Lewis, and P. R. Griffiths, "Anti-Stokes Raman spectrometry with 1064-nm excitation: an effective instrumental approach for field detection of explosives," Applied Spectroscopy, vol. 58, no. 4, pp. 420-427, 2004.

[21] M. L. Lewis, I. R. Lewis, and P. R. Griffiths, "Evaluation of a dispersive Raman spectrometer with a Ge array detector and a $1064 \mathrm{~nm}$ laser for the study of explosives," Vibrational Spectroscopy, vol. 38, no. 1-2, pp. 11-16, 2005.

[22] M. L. Lewis, I. R. Lewis, and P. R. Griffiths, "Raman spectrometry of explosives with a no-moving-parts fiber coupled spectrometer: a comparison of excitation wavelength," Vibrational Spectroscopy, vol. 38, no. 1-2, pp. 17-28, 2005.

[23] H. Owens, D. E. Battey, M. J. Pelletier, and J. B. Slater, "New spectroscopic instrument based on volume holographic optical elements," in Practical Holography IX, vol. 2406 of Proceedings of SPIE, pp. 260-267, February 1995.

[24] J. M. Tedesco, H. Owen, and B. J. Chang, "Detection system for Raman scattering employing holographic diffraction," US Pat, 5, 011, 284, 1991.

[25] J. Ramsey, S. Ranganathan, R. L. McCreery, and J. Zhao, "Performance comparisons of conventional and line-focused surface Raman spectrometers," Applied Spectroscopy, vol. 55, no. 6, pp. 767-773, 2001.

[26] J. M. Lerner, "Imaging spectrometer fundamentals for researchers in the biosciences-a tutorial," Cytometry A, vol. 69, no. 8, pp. 712-734, 2006.

[27] http://www.microscopyu.com/articles/formulas/formulasfielddepth.html.

[28] http://www.headwallphotonics.com/.

[29] J. Zhao and R. L. McCreery, "Multichannel FT-Raman spectroscopy: noise analysis and performance assessment," Applied Spectroscopy, vol. 51, no. 11, pp. 1687-1697, 1997.

[30] P. Vitek, E. M. A. Ali, H. G. M. Edwards, J. Jehlicka, R. Cox, and K. Page, "Evaluation of portable Raman spectrometer with $1064 \mathrm{~nm}$ excitation for geological and forensic applications," Spectrochimica Acta A, vol. 86, pp. 320-327, 2012.

[31] T. J. Johnson, Y.-F. Su, K. H. Jarman et al., "Demonstrated wavelength portability of Raman reference data for explosives and chemical detection," International Journal of Spectroscopy, vol. 2012, Article ID 297056, 11 pages, 2012.

[32] S. Moore, M. Schantz, and W. MacCrehan, "Characterization of three types of semtex (H, 1A, and 10)," Propellants, Explosives, Pyrotechnics, vol. 35, no. 6, pp. 540-549, 2010.
[33] R. Infante-Castillo, L. C. Pacheco-Londoño, and S. P. Hernández-Rivera, "Monitoring the $\alpha \rightarrow \beta$ solid-solid phase transition of RDX with Raman spectroscopy: a theoretical and experimental study," Journal of Molecular Structure, vol. 970, no. 1-3, pp. 51-58, 2010.

[34] P. Torres, L. Mercado, I. Cotte et al., "Vibrational spectroscopy study of $\beta$ and $\alpha$ RDX deposits," Journal of Physical Chemistry $B$, vol. 108, no. 26, pp. 8799-8805, 2004.

[35] I. G. Goldberg and J. A. Swift, "New insights into the metastable $\beta$ form of RDX," Crystal Growth \& Design, vol. 12, pp. 1040-11045, 2012.

[36] H. Oluseyi, A. Karcher, W. Kolbe et al., "Characterization and deployment of large format, fully depleted, back-illuminated, p-channel CCDs for precision astronomy," in Sensors, Systems, and Next-Generation Satellites VIII, vol. 5570 of Proceedings of SPIE, pp. 515-524, September 2004.

[37] M. S. Robbins, P. Mistry, and P. R. Jorden, "Detailed characterisation of a new large area CCD manufactured on high resistivity silicon," in Sensors, Cameras, and Systems for Industrial, Scientific, and Consumer Applications XII, vol. 7875 of Proceedings of SPIE, p. 876627, January 2011.

[38] K. F. Schrum, S. H. Ko, and D. Ben-Amotz, "Description and theory of a fiber-optic confocal and super-focal raman microspectrometer," Applied Spectroscopy, vol. 50, no. 9, pp. 11501155, 1996.

[39] K. A. Christensen and M. D. Morris, "Hyperspectral Raman microscopic imaging using Powell lens line illumination," Applied Spectroscopy, vol. 52, no. 9, pp. 1145-1147, 1998.

[40] D. S. Moore and R. J. Scharff, "Portable Raman explosives detection," Analytical and Bioanalytical Chemistry, vol. 393, no. 6-7, pp. 1571-1578, 2009. 


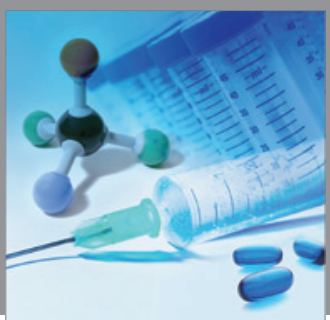

International Journal of

Medicinal Chemistry

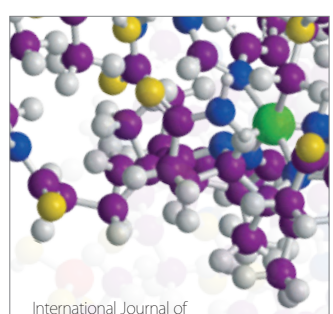

Carbohydrate Chemistry

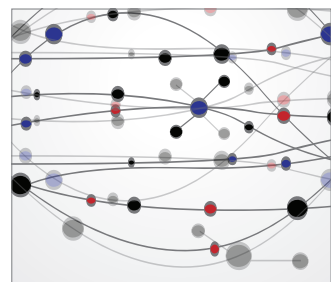

The Scientific World Journal
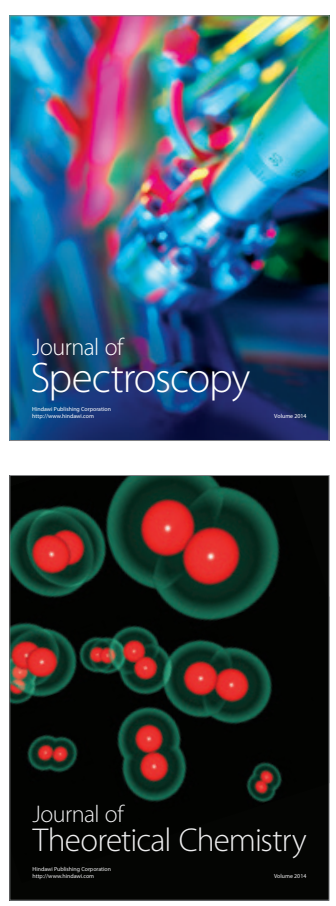
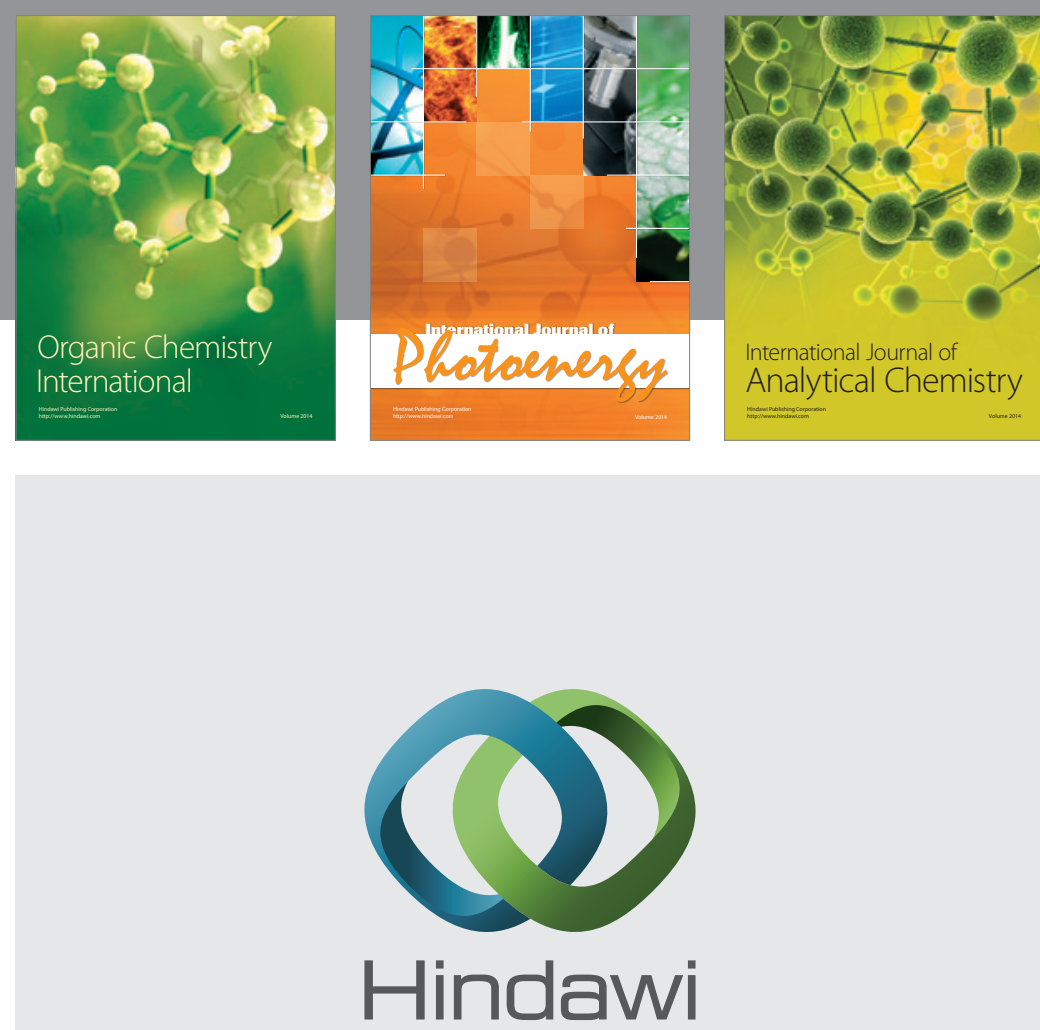

Submit your manuscripts at

http://www.hindawi.com
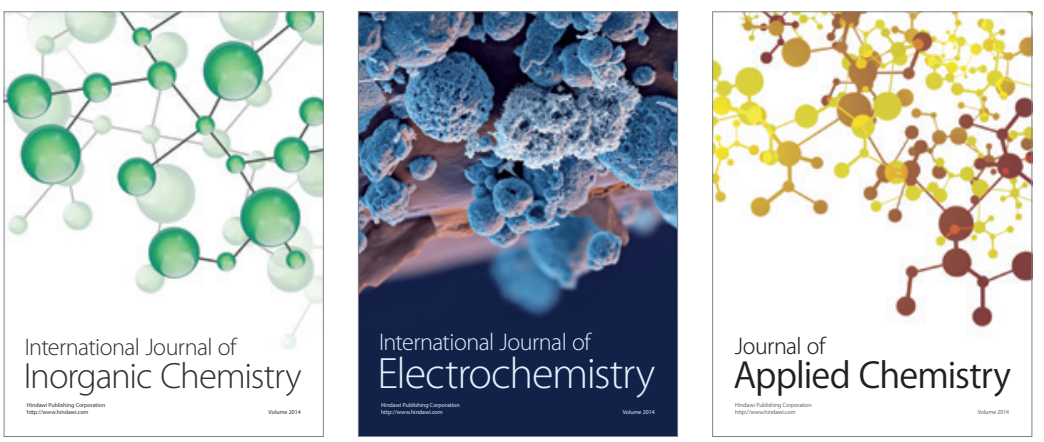

Journal of

Applied Chemistry

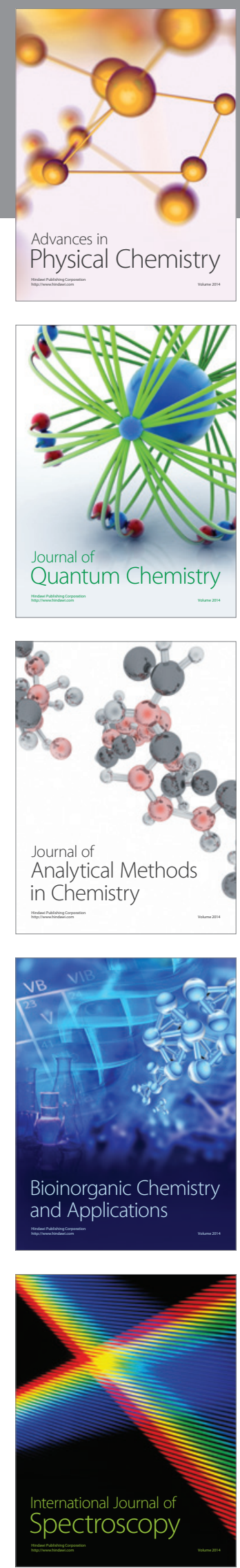